\title{
Longitudinal assessment of reactivity and affinity profile of anti-Jo1 autoantibodies to distinct HisRS domains and a splice variant in a cohort of patients with myositis and anti-synthetase syndrome
}

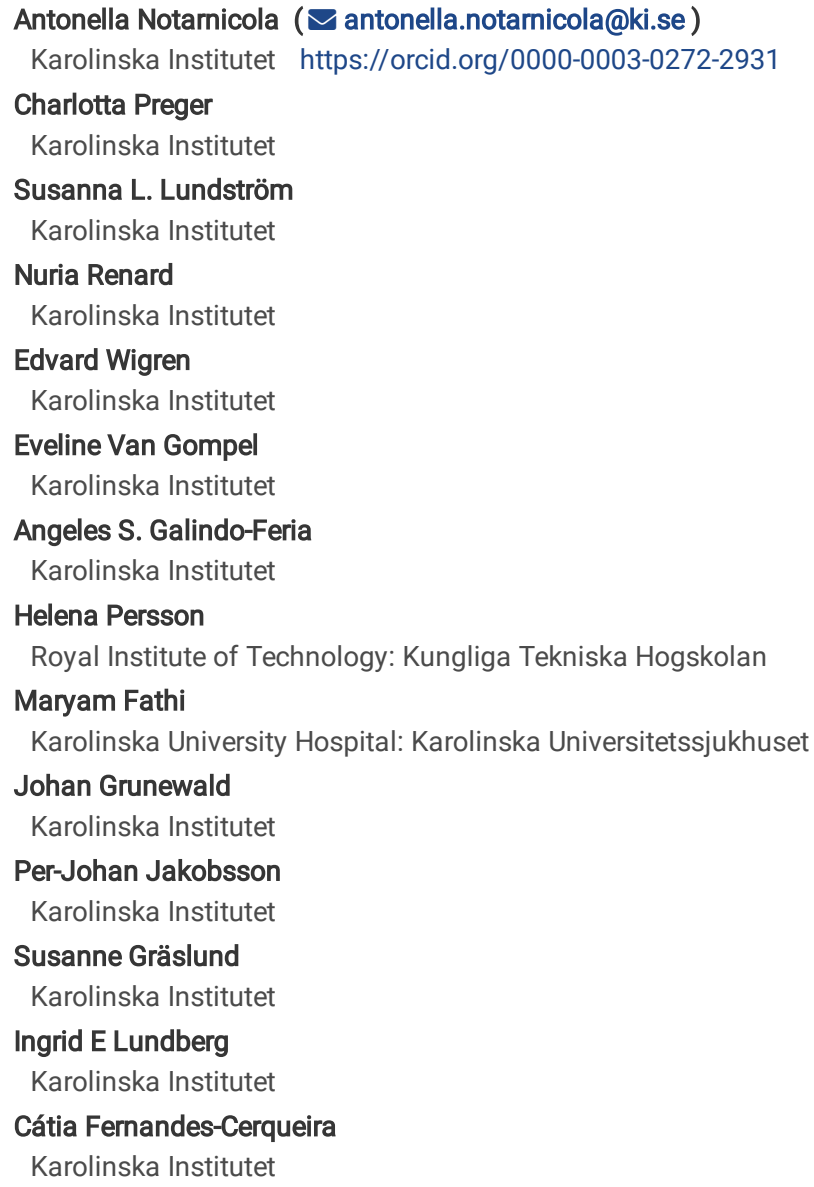

\section{Research Article}

Keywords: anti-J01, HisRS, longitudinal samples, ILD, autoantibodies, affinity, reactivity, BALF, idiopathic inflammatory myopathies, anti-synthetase syndrome Posted Date: July 8th, 2021

DOI: https://doi.org/10.21203/rs.3.rs-686543/v1

License: (9) (1) This work is licensed under a Creative Commons Attribution 4.0 International License. Read Full License

Version of Record: A version of this preprint was published at Arthritis Research \&amp; Therapy on March 2nd, 2022. See the published version at https://doi.org/10.1186/s13075-022-02745-6. 


\section{Abstract}

Background: To address the reactivity and affinity against histidyl-transfer RNA synthetase (HisRS) autoantigen of anti-Jo1 autoantibodies from serum and bronchoalveolar lavage fluid (BALF) in patients with idiopathic inflammatory myopathies/anti-synthetase syndrome (IIM/ASS). To investigate the associations between the reactivity profile and clinical data over time.

Methods: Samples and clinical data were obtained from: i) 25 anti-Jo $1^{+}$patients (19 sera with 16 longitudinal samples and 6 BALF/matching sera at diagnosis; ii) 29 anti-Jo1- patients (25 sera and 4 BALF/matching sera at diagnosis); iii) 27 age/gender-matched healthy controls (24 sera and 3 $\mathrm{BALF} /$ matching sera). Reactivity towards HisRS full-length (HisRS-FL), three HisRS domains (WHEP, antigen binding domain (ABD), and catalytic domain (CD)) and the HisRS splice variant (SV) was tested. Anti-Jo1 IgG reactivity was evaluated by ELISA and western blot using IgG purified from serum by affinity chromatography. In paired serum-BALF, anti-Jo1 IgG and IgA reactivity was analyzed by ELISA. Autoantibody affinity was measured by surface plasmon resonance using IgG purified from sera. Correlations between autoantibody reactivity and clinical data were evaluated at diagnosis and longitudinally.

Results: Anti-Jo1 IgG from serum and BALF bound HisRS-FL, WHEP, and SV with high reactivity at the time of diagnosis and recognized both conformationdependent and -independent HisRS epitopes. Anti-HisRS-FL IgG displayed high affinity early in the disease. At the time of IIM/ASS diagnosis, the highest autoantibody levels against HisRS-FL were found in patients ever developing interstitial lung disease (ILD) and arthritis, but with less skin involvement. Moreover, the reactivity of anti-WHEP IgG in BALF correlated with poor pulmonary function.

Levels of autoantibodies against HisRS-FL, -domains and -splice variant generally decreased over time. With some exceptions, longitudinal anti-HisRS-FL antibody levels changed in line with ILD activity.

Conclusion: High levels and high-affinity anti-Jo1 autoantibodies towards HisRS-FL were found early in disease in sera and BALF. In combination with the correlation of anti-HisRS-FL antibody levels with ILD and ILD activity in longitudinal samples as well as of anti-WHEP IgG in BALF with poor pulmonary function, this supports the hypothesis that the lung may have a role in the immune reaction in anti-Jo1 positive patients.

\section{Background}

Idiopathic inflammatory myopathies (IIM) are rare autoimmune, chronic inflammatory diseases associated with high mortality and morbidity (1, 2). A major IIM sub-group, termed anti-synthetase syndrome (ASS), affects skeletal muscle, lung, joints, and skin and is characterized by the presence of autoantibodies that target aminoacyl transfer(t) RNA synthetases (aaRS) (3). Anti-histidyl tRNA synthetase (HisRS) autoantibodies (anti-Jo1) are the most common anti-aaRS autoantibodies detected in $15-36 \%$ of IIM patients (4-6). Remarkably, up to $90 \%$ of IIM/ASS patients diagnosed with interstitial lung disease (ILD) have antiJo1 autoantibodies (7).

HisRS is a homodimeric protein composed of three domains, the WHEP domain located at the N-terminus, an internal catalytic domain (CD), and the anticodon binding domain (ABD) at the C-terminal end (Fig. 1A) (8). In 2012, a monomeric HisRS splice variant (SV) comprising the WHEP domain and the ABD (lacking the CD) was discovered (9). Later, an additional HisRS splice variant composed of the first 60 amino acids (WHEP domain itself) was described, and found to be overexpressed in the lung compared to other human tissues $(10,11)$. Both full-length HisRS (HisRS-FL) and the WHEP domain were shown, in vitro, to be secreted from the cytosol of different cell lines including lung and muscle cells into the extracellular environment (10). In addition, HisRS was detected in serum from patients with IIM/ASS and in healthy individuals (12). Interestingly, serum levels of HisRS protein were lower in patients with anti-Jo1 autoantibodies compared to patients with IIM/ASS without anti-Jo1 autoantibodies and healthy individuals (12). Critical work in previous studies has convincingly demonstrated that the anti-Jo1 response in myositis is directed towards several epitopes within the HisRS molecule, and particularly the WHEP domain (13-18). However, these studies were performed using linker mutagenesis and restriction enzymes, or linear peptide design and not complete protein domains mimicking naturally folded HisRS present inside cells and in circulation. When analyzing autoantibody reactivity against linear epitopes, as performed in previous studies, there is a large risk of missing the detection of conformational-dependent autoantibodies. The reactivity profile of anti-Jo1 antibodies against HisRS-FL, domains, and SV has so far only been assessed in sera and not in other biological samples such as the bronchoalveolar lavage fluid (BALF). Moreover, the anti-Jo1 response has previously been investigated in sera from anti-Jo1 positive patients and not on purified anti-Jo1 IgG. Testing the reactivity of purified anti-Jo1 antibodies will limit the influences of other molecules in the sera that could interfere with the antigen binding. In addition, only very limited data is available concerning the behavior of anti-Jo1 antibody levels during the disease course and in relation to the different clinical phenotypes and treatments.

The findings described in previous studies have raised several important questions. Firstly, could anti-HisRS autoimmunity be initiated towards a specific region of the protein e.g. WHEP domain which is highly expressed in the lungs (10), and during IIM/ASS disease course spread throughout the HisRS molecule? Secondly, acknowledging the strong association between ILD and the anti-Jo1 response in IIM/ASS (19), could anti-Jo1 autoantibodies targeting specific regions of HisRS be associated with distinct clinical phenotypes? Lastly, are anti-Jo1 autoantibodies in circulation recognizing the same HisRS epitopes as the autoantibodies found in the BALF of the lungs?

To address these questions, we extended previous epitope mapping studies to evaluate the reactivity profile of anti-Jo1 IgG and IgA from serum and BALF against HisRS-FL, the naturally occurring folded HisRS splice variant (SV), and separate HisRS domains (WHEP, CD and ABD). Additionally, we explored the association between the anti-Jo1 reactivity to the full-length protein, single domains and the splice variant of HisRS in relation to clinical manifestations in longitudinally collected serum samples and compared serum and BALF-derived anti-Jo1 autoantibodies collected at IIM/ASS diagnosis. To get a deeper understanding of the binding profile and the development of the anti-Jo1 autoantibodies, we also investigated the affinity of these only against HisRS-FL at the time of disease diagnosis. 


\section{Materials And Methods \\ Patient samples}

Stored sera from consecutive patients with IIM/ASS (19 anti-Jo $1^{+}$and 25 anti-Jo1 ${ }^{-}$, cohort 1, Table 1) attending the Rheumatology clinic at Karolinska University Hospital, Stockholm, Sweden were identified for IgG purification. Classification of IIM was made according to the Bohan and Peter criteria (20, 21). Griggs criteria (22) were applied for Inclusion Body Myositis (IBM). The diagnosis of ASS was based on the presence of anti-aaRS autoantibodies, plus one of the following features: ILD, myositis, arthritis, Raynaud's phenomenon, fever, or mechanic's hands (23). The first available serum sample in relation to IIM/ASS diagnosis was selected (median disease duration in Table 1). However, in three anti-Jo $1^{+}$and four anti-Jo $1^{-}$patients, the first available sera were collected at time points before diagnosis. Specifically, up to 3 months before diagnosis, median -1 month (25-75th percentiles -3 to -1$)$ for the anti-Jo $1^{+}$ group, and up to 21 months before diagnosis, median - 10 months (25-75th percentiles - 19.5 to -4.25$)$ for the anti-Jo $1^{-}$group. Longitudinal serum samples for IgG purification were available from 16 of the 19 anti-Jo $1^{+}$IIM/ASS patients up to 24 years after diagnosis (Supplementary Fig. 2). 
Table 1

Demographic data of cohort 1 at the time of first available serum sample*.

\begin{tabular}{|c|c|c|c|}
\hline & $\begin{array}{l}\text { IIM/ASS } \\
(n=44)\end{array}$ & $\begin{array}{l}\text { Anti-Jo1+ } \\
(n=19)\end{array}$ & $\begin{array}{l}\text { Anti-Jo1- } \\
(n=25)\end{array}$ \\
\hline Age, mean years (SD) & $57(13)$ & $52(14)$ & $61(12)^{a}$ \\
\hline Women, $\mathrm{n}(\%)$ & $24(55)$ & $9(47)$ & $15(60)$ \\
\hline Disease duration in months, median (25-75th percentiles) ${ }^{\star \star}$ & $0(0-1)$ & $1(0-10)$ & $0(0-1)$ \\
\hline Anti-synthetase syndrome (ASS), n (\%) & $28(64)$ & $19(100)$ & $9(36)^{b}$ \\
\hline \multicolumn{4}{|l|}{ Muscular manifestations, n ever (\%) } \\
\hline Muscle weakness (pathological MMT8 and/or FI-2) & $35(83)$ & $15(79)$ & $20(87)$ \\
\hline Muscle enzymes elevation (CK, LD, ASAT, ALAT) & $35(83)$ & $15(79)$ & $20(87)$ \\
\hline Muscle inflammatory infiltrates & $26(62)$ & $11(58)$ & $15(65)$ \\
\hline \multicolumn{4}{|l|}{ Extra-muscular manifestations, $\mathrm{n}$ ever (\%) } \\
\hline Interstitial Lung Disease (ILD) & $25(57)$ & $16(84)$ & $9(36)^{c}$ \\
\hline Skin rash ${ }^{\star * *}$ & $14(32)$ & $5(26)$ & $9(36)$ \\
\hline Arthritis & $18(41)$ & $11(58)$ & $7(28)$ \\
\hline Dysphagia & $9(21)$ & $3(16)$ & $6(24)$ \\
\hline Raynaud's phenomenon & $2(5)$ & $2(11)$ & $0(0)$ \\
\hline Smoking status, $n$ ever (\%) & $24(55)$ & $10(53)$ & $14(56)$ \\
\hline \multicolumn{4}{|l|}{ Laboratory tests } \\
\hline CK, median $\mu$ cat/L (25-75th percentiles) & $4.3(1.4-14.2)$ & $3.8(1.1-9.0)$ & $4.4(1.6-16.2)$ \\
\hline CRP, median $\mathrm{mg} / \mathrm{L}$ (25-75th percentiles) & $4.0(0.9-8.3)$ & $7.0(2.0-9.0)$ & $2.0(0.5-8.0)$ \\
\hline \multicolumn{4}{|l|}{ Autoantibodies } \\
\hline Positive anti-PL7, n (\%) & $2(5.1)$ & 0 & $2(8.3)$ \\
\hline Positive anti-PL12, n (\%) & $2(5.1)$ & 0 & $2(8.3)$ \\
\hline Positive anti-EJ, n (\%) & $1(2.6)$ & 0 & $1(4.2)$ \\
\hline Positive anti-OJ, n (\%) & $3(7.7)$ & 0 & $3(12.5)$ \\
\hline Positive anti-Mi-2, n (\%) & $3(7.9)$ & $1(7.1)$ & $2(8.3)$ \\
\hline Positive anti-SRP, n (\%) & $2(5.1)$ & 0 & $2(8.3)$ \\
\hline Positive anti-MDA5, n (\%) & $3(7.9)$ & 0 & $3(12.5)$ \\
\hline Positive anti-TIF1g, n (\%) & $3(7.9)$ & 0 & $3(12.5)$ \\
\hline Positive anti-SSA, n (\%) & $16(36.4)$ & $10(52.6)$ & $6(24.0)$ \\
\hline Positive anti-Ro52, n (\%) & $12(38.7)$ & $8(47.1)$ & $4(28.6)$ \\
\hline Positive anti-SSB, n (\%) & 0 & 0 & 0 \\
\hline
\end{tabular}

*First available serum samples collected: i) at diagnosis ( 0 months) sera was available from 6 anti-Jo $1^{+}$and 14 anti-Jo1' patients; ii) before diagnosis sera were available from 3 anti-Jo $1^{+}$and 4 anti-Jo $1^{-}$patients (median months [25-75th percentile], $-1[-3--1]$ and $-10[-19.5--4.25]$, respectively); iii) after

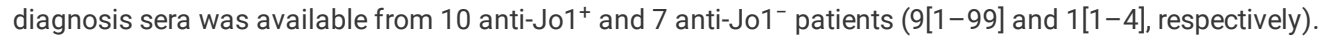

IIM, Idiopathic Inflammatory Myopathies; ASS, Anti-Synthetase Syndrome; CK, Creatinine kinase (reference values: 0.6-3.5 $\mu$ kat/L); CRP, C-reactive protein $(0-3 \mathrm{mg} / \mathrm{L})$; VAS, Visual Analogue Scale; MDDAT, Myositis Disease Activity Assessment Tool; HAQ, Health Assessment Questionnaire; MMT-8, Manual Muscle Testing.

1 treatment designates one of the following: methotrexate (Mtx), glucocorticoids (GC), intravenous immunoglobulin, or abatacept; 2 or 3 concomitant treatments designate all the possible following combinations: $G C+$ azathioprine (Aza), GC + cyclophosphamide, GC + Mtx, GC + mycophenolate mofetil (MMF), GC + rituximab, GC + cyclophosphamide + rituximab, GC + Mtx + rituximab, or GC + MMF + rituximab.

${ }^{* *}$ Disease duration was calculated based on month and year of clinical diagnosis; ${ }^{* \star}$ Skin rash features: Periungual erythema, mechanic's hand, Gottron's sign, Gottron's papules, V-sign, shawl sign, alopecia, erythroderma, periorbital edema, heliotrope rash.

${ }^{a} p=0.0236 ;{ }^{b} p<0.0001 ;{ }^{c} p=0.0020$ vs anti-Jo ${ }^{+}$(Mann-Whitney or Fisher's exact test). 


\begin{tabular}{|c|c|c|c|}
\hline & $\begin{array}{l}\text { IIM/ASS } \\
(n=44)\end{array}$ & $\begin{array}{l}\text { Anti-Jo1 } \\
(n=19)\end{array}$ & $\begin{array}{l}\text { Anti-Jo1- } \\
(n=25)\end{array}$ \\
\hline Positive anti-U1 RNP, n (\%) & $5(11.4)$ & $2(10.5)$ & $3(12.0)$ \\
\hline Positive anti-Ku, n (\%) & $1(2.5)$ & 0 & $1(4.0)$ \\
\hline Positive anti-PmScl, n (\%) & $2(4.9)$ & $1(6.3)$ & $1(4.0)$ \\
\hline Physician VAS, median (25-75th percentiles) & $40(25-60)$ & $45(32-60)$ & $40(17-50)$ \\
\hline Patient VAS, median (25-75th percentiles) & $40(16-69)$ & $44(19-70)$ & $32(11-68)$ \\
\hline HAQ (1-3), median (25-75th percentiles) & $0.88(0.00-1.50)$ & $0.75(0.19-1.25)$ & $1.00(0.00-1.63)$ \\
\hline MMT-8 (0-80), median (25-75th percentiles) & $78(67-80)$ & $79(77-80)$ & $75(64-80)$ \\
\hline Muscle activity score VAS, median (25-75th percentiles) & $15(0$ 37.5) & $4.5(0-35.5)$ & $15(0-37.5)$ \\
\hline MDAAT, median (25-75th percentiles) & $0.07(0.05-0.16)$ & $0.12(0.05-0.17)$ & $0.06(0.03-0.16)$ \\
\hline Extra-muscular activity, median (25-75th percentiles) & $32(15-40)$ & $40(11-43)$ & $24(16-34)$ \\
\hline \multicolumn{4}{|l|}{ Immunosuppressive (IS) treatment, n (\%) } \\
\hline No treatment & $10(26)$ & $4(25)$ & $6(26)$ \\
\hline 1 treatment & $10(26)$ & $2(13)$ & $8(35)$ \\
\hline \multirow[t]{2}{*}{2 or 3 concomitant treatments } & $19(49)$ & $10(63)$ & $9(39)$ \\
\hline & \multicolumn{3}{|c|}{ Healthy controls $(n=24)$} \\
\hline Age, mean years (SD) & \multicolumn{3}{|l|}{$59.3(13.0)$} \\
\hline Women, n (\%) & \multicolumn{3}{|l|}{12 of $24(50)$} \\
\hline \multicolumn{4}{|c|}{$\begin{array}{l}\text { *First available serum samples collected: i) at diagnosis ( } 0 \text { months) sera was available from } 6 \text { anti-Jo } 1^{+} \text {and } 14 \text { anti-Jo } 1^{-} \text {patients; ii) before diagnosis } \\
\text { sera were available from } 3 \text { anti-Jo } 1^{+} \text {and } 4 \text { anti-Jo } 1^{-} \text {patients (median months }[25-75 \text { th percentile], }-1[-3--1] \text { and }-10[-19.5--4.25] \text {, respectively); iii) aft } \\
\text { diagnosis sera was available from } 10 \text { anti-Jo } 1^{+} \text {and } 7 \text { anti-Jo } 1^{-} \text {patients }(9[1-99] \text { and } 1[1-4] \text {, respectively). }\end{array}$} \\
\hline \multicolumn{4}{|c|}{$\begin{array}{l}\text { IIM, Idiopathic Inflammatory Myopathies; ASS, Anti-Synthetase Syndrome; CK, Creatinine kinase (reference values: } 0.6-3.5 \mu \text { kat/L); CRP, C-reactive proteir } \\
\text { (0-3 mg/L); VAS, Visual Analogue Scale; MDDAT, Myositis Disease Activity Assessment Tool; HAQ, Health Assessment Questionnaire; MMT-8, Manual } \\
\text { Muscle Testing. }\end{array}$} \\
\hline \multicolumn{4}{|c|}{$\begin{array}{l}1 \text { treatment designates one of the following: methotrexate (Mtx), glucocorticoids (GC), intravenous immunoglobulin, or abatacept; } 2 \text { or } 3 \text { concomitant } \\
\text { treatments designate all the possible following combinations: } G C+\text { azathioprine (Aza), GC + cyclophosphamide, GC + Mtx, GC + mycophenolate mofetil } \\
\text { (MMF), GC + rituximab, GC + cyclophosphamide + rituximab, GC + Mtx + rituximab, or GC + MMF + rituximab. }\end{array}$} \\
\hline \multicolumn{4}{|c|}{$\begin{array}{l}{ }^{*} \text { Disease duration was calculated based on month and year of clinical diagnosis; }{ }^{* \star} \text { Skin rash features: Periungual erythema, mechanic's hand, Gottron's } \\
\text { sign, Gottron's papules, V-sign, shawl sign, alopecia, erythroderma, periorbital edema, heliotrope rash. }\end{array}$} \\
\hline
\end{tabular}

Matching BALF and serum collected at the time of diagnosis were available in the biobank from 10 additional IIM/ASS patients $\left(6\right.$ anti-Jo $1^{+}$and 4 anti-Jo1 ${ }^{-}$, Cohort 2, Supplementary Table 1).

Patients were defined as anti-Jo $1^{+}$if they had ever tested positive for anti-Jo1 antibodies by standardized immunoassays (immunoprecipitation, Line Blot or ELISA).

In cohort 1 and 2 , the mean age for the anti-Jo $1^{+}$IIM/ASS group was lower compared to anti-Jo1- IIM/ASS (54 vs 61, p = 0.0467). All anti-Jo $1^{+}$patients from cohort 1 and 2 (48\% women) were diagnosed with ASS, compared to $31 \%$ in anti-Jo1- $(p<0.0001)$ and $88 \%$ of anti-Jo $1^{+}$IIM/ASS had ILD in contrast to $34 \%$ in anti-Jo1- patients $(\mathrm{p}<0.0001)$. Demographics are presented in Table 1 and Supplementary Table 1. Serum samples from healthy control individuals $(\mathrm{HC})$ were selected to match IIM/ASS patients for age and gender (mean age 59 years, 50\% women).

\section{Definition of clinical, laboratory and disease activity data}

Signs of muscular involvement such as muscle weakness based on pathological manual muscle test-8 (MMT-8) and/or myositis functional index-2 (FI-2) (24), muscle enzymes elevation (creatine kinase (CK), lactate dehydrogenase (LD), aspartate aminotransferase (ASAT), alanine aminotransferase (ALAT)), and inflammatory infiltrates in muscle biopsies, present at any time during disease course were recorded.

Extra-muscular manifestations such as ILD, arthritis, skin rash (periungual erythema, mechanic's hand, Gottron's sign, Gottron's papules, V-sign, shawl sign, alopecia, erythroderma, periorbital edema, heliotrope rash), Raynaud's phenomenon, and dysphagia present at any time during disease course were recorded. Smoking status was defined as never/ever smoker. 
Diagnosis of ILD was based on the American Thoracic Society criteria (25). All patients were screened with pulmonary function tests and high-resolution computer tomography (HRCT) of the lungs to confirm or exclude the presence of ILD with the exception of four anti-Jo1 ${ }^{-}$patients in cohort 1 who only underwent lung x-ray. In patients with ILD, spirometry test results (forced vital capacity (FVC), total lung capacity (TLC) and diffusion lung capacity of carbon monoxide (DLCO)) and HRCT data were retrieved at the time of each serum and BALF sample when available. The pattern of ILD (non-specific interstitial pneumonia (NSIP), usual interstitial pneumonia (UIP) and organizing pneumonia (OP)) was retrieved. By considering the change of spirometry test results and HRCT from time of diagnosis to the last available investigation (not always corresponding to the last longitudinal sample included in the analysis) a global ILD outcome was assigned as improvement, stable or progression.

Longitudinal disease activity was assessed by collecting variables of the IMACS (International Myositis Assessment \& Clinical Studies group) disease activity core set measures (24) and by calculating the total improvement score according to the IMACS response criteria. For details, please see Supplementary Methods.

In patients who developed arthritis, disease activity score-28 (DAS-28) was recorded close to the date of each serum sample. Comparing the first and last DAS28 , the response was defined according to EULAR response criteria (26). In case the patient just had one available serum sample, the response was calculated by comparing the DAS-28 at the date of sample and the last DAS-28 available in medical records and/or SweMyonet/Euromyositis registers after the date of sample. Information on treatment at the time of sampling was collected. Administration of rituximab before each included serum sample was recorded (Table 1, Fig. 3B and Supplementary Fig. 6).

\section{ELISA and western blot analysis}

Biotinylated HisRS variants and control proteins utilized for ELISA, western blot (WB), and affinity measurements were generated as previously described (27). Information on antigen ID, molecular weight, and amino acid coverage of the proteins is depicted in Fig. 1A, Supplementary Table 2, and Supplementary Fig. 1. To avoid interference of other serum factors, IgG was purified from serum as described before $(28,29)$. More information is found in Supplementary Fig. 2.

ELISA and western blot experiments to evaluate the reactivity of serum and BALF-derived anti-Jo1 autoantibodies (IgG and IgA) against HisRS-FL, HisRS domains, and splice variant are described in Supplementary Methods. ELISA was executed in: i) IgG purified from serum of 44 IIM/ASS and 24 HC individuals (anti-Jo1 IgG detection); and ii) 13 BALF and 13 matched-sera, 10 from IIM/ASS patients and 3 from HC. Total IgG, total IgA, anti-Jo1 IgG, and anti-Jo1 IgA were measured both in undiluted BALF and 1:500 diluted serum. The biotinylated variants of HisRS were added to streptavidin coated plates in high excess compared to the amount of antibody tested to avoid the effects of different molar concentration of antigen due to the different molecular weight of HisRS versions.

Anti-Jo1 lgG levels in serum (ng/mL) were calculated based on a standard curve generated from anti-Jo1 lgG enriched from a sera pool of 38 IIM/ASS patients (Supplementary Fig. 3B). The antibody levels were measured in the linear range between 5 and $100 \mathrm{ng} / \mathrm{mL}$. These specific anti-Jo1 lgG were also enriched from serum by affinity chromatography as previously described, followed by a HisRS chromatography column $(28,29)($ prepared in house, Supplementary Methods).

Autoantibody reactivity in IgG purified from serum of 19 anti-Jo1 ${ }^{+}$patients, 2 anti-Jo1- patients and 3 HC was also tested by WB (Fig. 1D, Supplementary Fig. 5B).

\section{Surface plasmon resonance}

Affinity measurements of serum-derived IgG to HisRS-FL, close to diagnosis (between $-0.25-0$ years), from the 19 anti-Jo $1^{+}$patients were performed using surface plasmon resonance (SPR). The measurements were carried out using the Biacore T200 biosensor instrument (Cytiva), single cycle kinetics mode, and the Biacore T200 evaluation 3.1 software (Cytiva) was used for analyses. The measurements were done by capturing total IgG on the surface and flowing HisRS over the system to avoid measuring the avidity from the mix of polyclonal anti-Jo1 antibodies, for more details see Supplementary Methods.

\section{Statistical analysis}

Continuous variables with normal distribution were presented as means with standard deviations (SD), while variables that violated normality were presented as medians with 25-75th percentiles [25-75th ]. Comparison of categorical variables was performed using Fisher's exact test or Chi-square test, when appropriate. Kruskal-Wallis, Friedman's (correction for multiple comparisons by Dunn's test), or Mann-Whitney tests were employed when quantitative variables were compared among all groups or between two groups, respectively. Correlations between anti-Jo1 lgG/lgA reactivity levels and clinical data were performed using Spearman's rank coefficient correlation. $\mathrm{P}<0.05$ denotes a significant difference. Data analysis was done using GraphPad Prism version 8 (La Jolla, USA). Multivariate modelling using Principal Component Analysis (PCA) and Orthogonal projections to latent structures discrimination analysis (OPLS-DA) was performed using SIMCA 15.0 (Umetrics, Sweden) following mean centering, log transformation and UV scaling. Model performance was reported as the cumulative correlation $\mathrm{R}^{2} \mathrm{X}[\mathrm{cum}]$, and predictive performance - as $\mathrm{Q}^{2}$ [cum] based on seven-fold cross validation.

\section{Results}

\section{Reactivity profile against HisRS of serum and BALF-derived anti-Jo1 autoantibodies}

\subsection{Anti-Jo1 reactivity of IgG purified from the first available serum sample}


Anti-Jo1 reactivity in total IgG purified from serum was evaluated by ELISA against HisRS (HisRS-FL), one HisRS splice variant (SV), and three HisRS domains (WHEP, CD and ABD), (Fig. 1). In the first available serum sample, median 1 month [0-13] post-diagnosis, total IgG from anti-Jo ${ }^{+}$patients displayed stronger reactivity against the HisRS-FL and the WHEP domain, in comparison with the CD and ABD, and splice variant SV (Fig. 1B and 1C, Supplementary Fig. 5A). The ELISA results were confirmed by WB (Fig. 1D, Supplementary Fig. 5B). Sixteen of 19 anti-Jo $1^{+}$patients showed reactivity to all HisRS antigens (with different degree of binding). One patient (P4) presented exclusive binding to ABD and SV by ELISA and WB (Fig. 1B and 1D) but reactivity to HisRS-FL was only detected by WB (Fig. 1D). Two patients (P1, P13), belonging to the group of anti-Jo1 positive patients with no evidence of ILD, presented no reactivity against any of the HisRS antigens by ELISA and WB (Fig. 1, Supplementary Fig. 5). Together, the ELISA and WB results confirm that anti-Jo1 antibodies recognize both conformation-dependent (ELISA) and -independent epitopes (WB), with a strong preference for the WHEP region. Anti-Jo1 ${ }^{-}$and HC did not show reactivity towards HisRS-FL or any of the HisRS-variant/domains (Fig. 1, Supplementary Fig. 5).

\subsection{Anti-Jo1 reactivity of IgG and IgA present in BALF and matched serum samples}

In BALF, anti-Jo1 IgG and IgA reactivity was registered against HisRS-FL as well as the different HisRS domains and splice variant with the strongest reactivity against HisRS-FL and SV in anti-Jo1+ IIM/ASS patients (Fig. 2A and 2B). Similarly, in serum samples collected at the same time as the BALF, the highest IgG and IgA reactivity was found against HisRS-FL (Fig. 2C, 2D). In anti-Jo $1^{+} \mathrm{IIM} / \mathrm{ASS}$ patients, no anti-Jo1 autoantibody enrichment ( $p>0.05$ ) could be found in BALF in comparison to paired serum samples, for either $\lg \mathrm{G}$ or $\lg \mathrm{A}$ (Supplementary Fig. 4B, 4E).

BALF and paired sera from age/gender-matched $\mathrm{HC}$ and clinically diagnosed anti-Jo1` patients did not display IgG or IgA reactivity against HisRS-FL, domains or -splice variant (Supplementary Fig. 4).

\subsection{Anti-Jo1 reactivity of IgG purified from serum of anti-Jo1 ${ }^{+}$patients collected longitudinally}

Longitudinal serum samples for IgG purification were available from 16/19 anti-Jo1+ IIM/ASS patients. The highest reactivity levels of anti-HisRS-FL and antiWHEP IgG were recorded at the time of diagnosis (median 97 and $81 \mathrm{ng} / \mathrm{mL}$, respectively), in comparison to anti-CD, anti-ABD, and anti-SV IgG (median 66,54 , and $50 \mathrm{ng} / \mathrm{mL}$, respectively) (Fig. 3A). Similar median IgG reactivity levels against HisRS were detected in the three anti-Jo $1^{+}$serum samples collected before diagnosis (median $100 \mathrm{ng} / \mathrm{mL}$ ). In 10 out of these 16 patients, IgG reactivity against the majority of HisRS antigens was lower at the end of the follow-up compared to the one registered close to diagnosis, although levels fluctuated through the disease course (Fig. 3A-B, Supplementary Figs. 6 and 7). However, three years after diagnosis, median anti-HisRS-FL IgG reactivity levels were still almost as high as our limit of detection (median of $92 \mathrm{ng} / \mathrm{mL}$ ), while the reactivity against WHEP and SV registered a decrease (median levels below limit of detection, Fig. $3 A$ ). The reactivity levels against CD and $A B D$ decreased between 7 to 2 -fold already one year after diagnosis (median of 10 and 22 ng/mL, respectively, Fig. 3A), remaining low thereafter (Fig. $3 \mathrm{~A}$ ).

The median antibody concentrations against the different HisRS antigens fluctuated over time and, in general, changed simultaneously. By following the longitudinal levels of anti-HisRS-FL antibodies, we noticed that the anti-HisRS-FL levels changed consistently with lung disease activity. In P11, P16, P17, P9, P10, P14, and P15 improvement or stabilization of ILD was registered when anti-HisRS-FL levels were lower than the levels recorded at the time of diagnosis (Fig. 3B, Supplementary Fig. 6). Accordingly, anti-HisRS-FL levels in P2 and P6 (Fig. 3B) increased in parallel to ILD progression. However, we also observed exceptions to this trend. For instance, in P3 and P5, ILD improved or didn't worsen respectively, despite the increase or the persistently high levels of antiHisRS-FL antibodies, respectively (Supplementary Fig. 6). On the other hand, in P8 and P12, ILD progressed despite the decrease of anti-HisRS-FL levels (Supplementary Fig. 6).

Data on DAS-28 at the different longitudinal time points were only available for few patients, thus not allowing to describe any significant result.

\subsection{Effects of rituximab}

Rituximab was administered in 9 of the 16 anti-Jo1 ${ }^{+}$patients. In two patients (P11 and P12, Fig. 3B, Supplementary Fig. 6) autoantibody levels against HisRS variant/domains decreased after rituximab, whereas in three patients (P1, P7 and P16, Fig. 3B, Supplementary Fig. 6), the antibody levels remained stable. In one patient (P6, Fig. 3B) we observed a switch in the reactivity after rituximab treatment, whereby anti-HisRS-FL, -WHEP, and -SV IgG levels increased and anti$C D$ and -ABD IgG decreased. Anti-HisRS-FL IgG levels also increased in patient P3 (Supplementary Fig. 6) after rituximab, while reactivity to the other HisRS variant/domains decreased. Samples collected after the administration of Rituximab were not available for P10 and P13.

\section{Correlations between clinical data and reactivity profile}

\subsection{Reactivity of anti-Jo1+ $\mathrm{IgG}$ purified from first available serum in relation to clinical data}

Considering the autoantibody levels targeting HisRS-FL (calculated based on the anti-Jo1 lgG standard curve, Supplementary Fig. 3B) in the first available sample close to the time of diagnosis, patients were stratified into low to moderate $(n=8,0.5-100 \mathrm{ng} / \mathrm{mL})$ or high anti-HisRS-FL reactivity $(n=11,>100$ ng/mL, Fig. 4, Supplementary Table 3).

In patients with high levels of anti-HisRS-FL antibodies, the median value of muscle disease activity score at diagnosis was 0 compared to 19 and 15 in patients with low to moderate or no anti-HisRS-FL autoantibodies, respectively, although differences did not reach statistical significance. MMT-8 scores and CK values at diagnosis as well as frequency of muscle weakness, muscle enzyme elevation and inflammatory infiltrates in muscle biopsies ever reported during the disease course did not statistically differ between those with low to moderate or high anti-HisRS-FL reactivity.

Page $7 / 19$ 
Anti-Jo $1^{+}$IIM/ASS patients with high anti-HisRS-FL antibody levels were more likely to be diagnosed with ILD, ever through the disease course (100\% compared to $63 \%$ for the anti-Jo ${ }^{+}$patient group with low to moderate anti-HisRS-FL IgG levels and $36 \%$ for anti-Jo $1^{-}$group, $p<0.05$, Fig. 4 A, Supplementary Table 3). ILD was present already at diagnosis in all anti-Jo $1^{+}$patients with reported lung manifestations. The pulmonary function (median values of FVC, TLC) in the low to moderate anti-HisRS-FL reactivity group was significantly lower compared to both anti-Jo ${ }^{+}{ }^{+}$with high anti-HisRS-FL levels and anti-Jo1 ${ }^{-}$ $(51 \%, 67 \%$ and $81 \%$ for FVC, and $54 \%, 70 \%$ and $76 \%$ for TLC, in respective groups $p<0.05$, Supplementary Table 3 ). Noteworthy, significantly more smokers were observed in the anti-Jo $1^{+}$group with low to moderate antibody levels (88\%) compared to those with high anti-HisRS-FL IgG titers $(27 \% \mathrm{p}<0.05$, Fig. $4 \mathrm{D})$.

The group with high anti-HisRS-FL antibody levels presented a higher percentage of arthritis $(73 \%)$ in comparison to the low to moderate, and negative subgroups ( $38 \%$ and $28 \%$, respectively, p $<0.05$, Fig. 4B, Supplementary Table 3 ). Anti-Jo ${ }^{+}$patients with low to moderate levels anti-HisRS-FL IgG levels, similarly to anti-Jo ${ }^{-}$patients, were more frequently diagnosed with skin manifestations $(38 \%, 36 \%$, and $18 \%$ for low to moderate, negative, and high anti-HisRS-FL response, respectively, p >0.05, Fig. 4C, Supplementary Table 3).

The presence of anti-Ro52 antibodies was higher in the anti-Jo $1^{+}$group with the high levels of anti-HisRS-FL antibodies, compared to low to moderate antiHisRS-FL and anti-Jo $1^{-}$groups (64\%, 17\%, 29\%, p > 0.05, Fig. 4E, Supplementary Table 3). The analysis of outcome measures such as total improvement score, global ILD outcome, and EULAR DAS-28 response did not show any significant difference between patients with low to moderate and high anti-HisRSFL levels.

Stratification based on high/low anti-WHEP, -CD, -ABD, and -SV IgG levels at time of diagnosis largely reflected the observations described for HisRS-FL (data not shown).

2.2 Reactivity of BALF IgG/IgA close to IIM/ASS diagnosis in relation to pulmonary function and BALF cellular content data

BALF levels of IgG anti-WHEP and anti-CD correlated negatively with several pulmonary function measures (VC, FVC, TLC, and FEV1, p < 0.05 r $>-0.8810$,

Table 2). Anti-HisRS-FL IgA correlated negatively with FEV1 $(p<0.05 r>-0.7785)$, and anti-SV IgA correlated positively with FEV1_VC ratio ( $p=0.044 r=-0.8407)$. 
Table 2

Correlations between clinical data and levels of anti-Jo1 IgG and IgA autoantibodies from BALF.

\begin{tabular}{|c|c|c|c|c|c|c|c|c|c|c|c|c|c|}
\hline \multirow{2}{*}{$\begin{array}{l}\text { Anti-Jo1 in } \\
\text { BALF } \\
\begin{array}{l}\text { Spearman } \\
\text { correlation }\end{array}\end{array}$} & \multicolumn{2}{|c|}{ Anti-HisRS-FL IgG } & \multicolumn{2}{|c|}{ Anti-HisRS-FL IgA } & \multicolumn{2}{|c|}{ Anti-WHEP IgG } & \multicolumn{2}{|c|}{ Anti-CD IgG } & \multirow{2}{*}{$\begin{array}{l}\text { Anti-ABD } \\
\text { lgG } \\
\text { r }\end{array}$} & \multicolumn{3}{|c|}{ Anti-SV IgG } & \multirow{2}{*}{$\begin{array}{l}\text { Anti-SV lg } \\
\mathrm{r}\end{array}$} \\
\hline & $\mathbf{r}$ & $\mathbf{P}$ & $\mathbf{r}$ & $\mathbf{p}$ & $\mathbf{r}$ & $\mathbf{p}$ & $\mathbf{r}$ & $p$ & & p & $\mathbf{r}$ & $p$ & \\
\hline VC $\%$ & -0.2143 & 0.6615 & -0.6786 & 0.1095 & -0.8810 & 0.0072 & -0.8929 & 0.0123 & -0.1429 & 0.7825 & -0.6071 & 0.1667 & -0.6071 \\
\hline FVC \% & -0.9000 & 0.0833 & -0.8000 & 0.1333 & -1.0000 & 0.0167 & -1.000 & 0.0167 & -0.3000 & 0.6833 & -0.9000 & 0.0833 & -0.2052 \\
\hline TLC \% & -0.4524 & 0.2675 & -0.6429 & 0.0962 & -0.8810 & 0.0072 & -0.8810 & 0.0072 & -0.0714 & 0.8820 & -0.6667 & 0.0831 & -0.2755 \\
\hline FEV1 \% & -0.2515 & 0.5495 & -0.7785 & 0.0301 & -0.8982 & 0.0049 & -0.9581 & 0.0007 & -0.1916 & 0.6479 & -0.7066 & 0.0591 & -0.6627 \\
\hline DLCO \% & -0.2143 & 0.6615 & -0.6071 & 0.1667 & -0.7143 & 0.0881 & -0.7500 & 0.0663 & -0.3571 & 0.4444 & -0.6071 & 0.1667 & -0.7143 \\
\hline $\begin{array}{l}\text { FEV1_VC } \\
\text { ratio }\end{array}$ & -0.2609 & 0.5889 & 0.0290 & 0.9944 & 0.4058 & 0.4444 & 0.4638 & 0.3944 & 0.7827 & 0.0722 & 0.4638 & 0.3944 & 0.8407 \\
\hline $\begin{array}{l}\text { Cell } \\
\text { concentration } \\
\text { x } 10 \mathrm{E} 6 / \text { liter }\end{array}$ & 0.5879 & 0.0806 & 0.9515 & 0.0001 & 0.7697 & 0.0126 & 0.8182 & 0.0058 & 0.6121 & 0.0667 & 0.6485 & 0.0490 & 0.8268 \\
\hline $\begin{array}{l}\text { Alveolar } \\
\text { macrophages } \\
\%\end{array}$ & -0.5030 & 0.1440 & -0.7576 & 0.0149 & -0.7455 & 0.0174 & -0.7939 & 0.0088 & -0.2121 & 0.5603 & -0.6242 & 0.0603 & -0.5106 \\
\hline $\begin{array}{l}\text { Lymphocytes } \\
\%\end{array}$ & 0.1273 & 0.7330 & 0.3939 & 0.2632 & 0.2485 & 0.4918 & 0.3697 & 0.2957 & -0.0424 & 0.9184 & 0.1152 & 0.7589 & 0.2614 \\
\hline Neutrophils \% & 0.5854 & 0.0800 & 0.1281 & 0.7251 & 0.5610 & 0.0959 & 0.3537 & 0.3145 & -0.2439 & 0.4945 & 0.5122 & 0.1331 & -0.2966 \\
\hline $\begin{array}{l}\text { Eosinophils } \\
\%\end{array}$ & 0.5671 & 0.0922 & 0.5183 & 0.1287 & 0.7866 & 0.0094 & 0.7195 & 0.0231 & 0.3049 & 0.3883 & 0.7744 & 0.0115 & 0.3670 \\
\hline Basophils \% & 0.06920 & 0.8444 & 0.2076 & 0.5778 & 0.2422 & 0.4889 & 0.3200 & 0.3778 & 0.7006 & 0.0222 & 0.3546 & 0.3333 & 0.6377 \\
\hline Mast cells* & 0.52288 & 0.1247 & 0.5957 & 0.0745 & 0.5897 & 0.0774 & 0.5836 & 0.0818 & 0.5228 & 0.1247 & 0.6261 & 0.0577 & 0.5793 \\
\hline $\begin{array}{l}\text { CD4:CD8 } \\
\text { ratio }\end{array}$ & -0.5532 & 0.1011 & -0.9119 & 0.0006 & -0.6201 & 0.0611 & -0.6809 & 0.0350 & -0.1702 & 0.6378 & -0.4134 & 0.2353 & -0.5000 \\
\hline \multicolumn{14}{|l|}{$\begin{array}{l}p<0.05 \\
\text { Significant } \\
\text { correlation } \\
\text { for lgG }\end{array}$} \\
\hline $\begin{array}{l}p<0.05 \\
\text { Significant } \\
\text { correlation } \\
\text { for } \lg A\end{array}$ & & & & & & & & & & & & & \\
\hline
\end{tabular}

*Number of cells per high power field.

BALF, bronchoalveolar lavage fluid; VC, vital capacity; FVC, forced vital capacity; TLC, total lung capacity FEV1, Forced expiratory volume in 1 second; DLCO, diffusion lung capacity for carbon monoxide.

Anti-WHEP and anti-CD IgG levels correlated positively with the BALF's cell concentration and the number of eosinophils $(p<0.05 r>0.7195)$. Both the antiHisRS-FL IgA and anti-SV IgA correlated positively with the cell concentration in BALF $(p<0.05 r>0.7697)$. Anti-ABD IgG correlated positively only with the number of basophils $(p<0.05 r=0.7006)$. A negative correlation was observed between anti-HisRS-FL IgA and anti-CD IgG and alveolar macrophages and CD4:CD8 ratio $(p<0.05 r>-0.6809$, Table 2, Supplementary Table 1). Similarly, anti-WHEP IgG correlated negatively with alveolar macrophages $(p=0.017$ $r>-0.7455)$. Since the IgA reactivity in BALF against WHEP, CD and ABD domains was very low, correlations were performed only with anti-HisRS-FL and antiSV-IgA (Fig. 2B, Table 2).

\section{Multivariate data analysis}

Multivariate data analysis was performed to identify correlations in the anti-HisRS reactivity profile and to obtain information on how this profile correlated with other clinical factors. Two types of principal component analysis (PCA) models were created: 1) including only the anti-HisRS reactivity data (described in Supplementary Results) and 2) including the anti-HisRS reactivity data combined with all other available information as described below.

Anti-HisRS-FL reactivity data correlated strongly with ILD ${ }^{+}$and anti-Jo $1^{+}$autoantibody status. Also, ASS diagnosis, presence of MSAs, anti-SSA antibodies, and arthritis correlated with anti-Jo $1^{+}$and ILD ${ }^{+}$patients in cohort 1 (Fig. 5A, 5B). SSA and Ro52 antibodies were detected in ten and eight anti-Jo $1^{+}$patients, respectively (in total, $80 \%$ of the SSA ${ }^{+}$were Ro52 ${ }^{+}$). We observed that ILD negative patients $(n=19)$, independently of anti-Jo1 status, correlated negatively with anti-HisRS-FL and anti-WHEP reactivity. In cohort 2, which included fewer patients but where we had more information on pulmonary status, both the antiHisRS-FL reactivity data from IgG and IgA as well as eosinophils and mast cells correlated strongly with anti-Jo $1^{+}$and ILD ${ }^{+}$status (Fig. 5C, 5D). Inversely, higher levels of VC, FEV1, TLC, FVC, DLCO, CD4:CD8 and macrophages correlated prominently with anti-Jo1 ${ }^{-}$and ILD ${ }^{-}$. 


\section{Affinity profile of anti-Jo1 + IgG purified from serum}

The binding profiles of serum-derived IgG to HisRS-FL, close to diagnosis, from the 19 anti-Jo1 ${ }^{+}$patients were analyzed using surface plasmon resonance (SPR). Average kinetic constants could be determined for 14 of the patients and in all cases high average affinity profiles were observed (calculated Ave $K_{D}$ close to $1 \mathrm{nM}$ ). A selection of representative sensorgrams is showed in Fig. 6. IgG from patients P1, P4 and P13 did not show any binding to HisRS-FL in SPR, confirming the results from ELISA and WB, and P6 and P10 displayed too low responses for determination of kinetic constants. The remaining 14 patients could be divided into two groups based on the average affinity profile: one group $(n=7)$ with a more biphasic off-rate (Fig. 6 , as exemplified by P9), and another group ( $n=7)$ with a slower and more homogenous off-rate (Fig. 6, as exemplified by P5 and P17). Detailed analysis of the binding and fitting of the interaction to a suitable model is complicated due to several factors. Therefore, to distinguish the average values reported herein from traditionally reported affinity $\left(K_{D}\right)$ and dissociation rate constants $\left(k_{d}\right)$, we opted to use the nomenclature ${ }^{A v e} K_{D}$ and ${ }^{A v e} k_{d}$.

\section{Discussion}

In the current study we sought to understand 1) the response displayed by purified IgG anti-Jo1 autoantibodies derived from serum towards HisRS-FL, one HisRS splice variant and single HisRS domains as well as IgG and IgA reactivity in paired serum and BALF towards the same protein variants; 2) possible associations between clinical manifestations and the pattern of anti-HisRS reactivity in circulation and in BALF both at the time of diagnosis and during the disease course; and 3) the affinity profile of anti-Jo1 autoantibodies against HisRS.

In our study we could demonstrate that purified IgG anti-Jo1 autoantibodies in sera from a time close to diagnosis of IIM/ASS exhibited high and multiple reactivities against the HisRS-FL, splice variant and domains, with a particularly strong reactivity against the WHEP domain and the HisRS-FL. A similar reactivity pattern to the different HisRS variants was observed in most anti-Jo1 positive patients. The ELISA results were confirmed by WB indicating that antiJo1 antibodies recognize both conformation-dependent (ELISA) and -independent epitopes (WB). In BALF the highest reactivity of both IgG and IgA anti-Jo1 autoantibodies was also directed towards the HisRS-FL and when compared to matched serum samples there were no signs of anti-Jo1 autoantibody enrichment in BALF.

Despite the overall similar antibody reactivity to the versions of the HisRS antigen seen in our patient cohort, we observed differences in clinical manifestations associated with the different levels of reactivities to the HisRS-FL antigen at the time of diagnosis. Thus, patients with high IgG serum levels towards HisRS-FL at diagnosis were more likely to ever present with ILD and arthritis, but less likely to have skin rash compared to patients with low to moderate anti-HisRS-FL IgG levels or anti-Jo1 negative. Furthermore, IgG anti-WHEP reactivity in BALF correlated with poor pulmonary function. These observations were further strengthened by applying an unbiased multivariate statistical analysis. The significant correlation between antibody reactivity (especially anti-WHEP) in the BALF at diagnosis and poor pulmonary function together with BALF inflammatory content (e.g. eosinophils, alveolar macrophages, and CD4/CD8 ratio), supports our hypothesis of an association between autoantibody reactivity towards the WHEP domain and lung involvement in IIM/ASS.

Anti-Ro52 antibodies, which have been reported to be associated with more severe ILD when present in serum together with anti-Jo1 antibodies (30-32), were more prevalent in the group of patients with higher anti-HisRS-FL reactivity. An unexpected finding in our study was that patients with low to moderate antiHisRS-FL reactivity presented with worse pulmonary function (lower FEV and TLC\%). Maybe this could be attributed to the significantly higher proportion of patients being ever smokers in this sub-group.

In the IgG purified from sera, the highest anti-HisRS reactivity levels were recorded before and at the time of diagnosis, and generally decreasing thereafter. In addition, IgG against HisRS-FL displayed high affinity already at diagnosis. The reactivity to HisRS-FL remained high, up to three years post-diagnosis, whereas the antibody response to the splice variant and HisRS domains decreased within the first year after diagnosis. This observation could explain why a consistent group of patients with IIM/ASS do not enter remission despite immunosuppressive treatment. Thanks to the access to longitudinal serum samples and clinical data collected at the same time points, we could observe that, despite some exceptions, reactivity levels towards HisRS-FL changed over time consistently with the degree of lung disease activity. In fact, longitudinal levels of anti-HisRS-FL increased in line with ILD progression and decreased when recording an improvement of ILD. Taken into consideration the low number of anti-Jo1 positive patients, this might suggest that anti-Jo1 antibodies could serve not only as diagnostic marker of ASS, but also supports a role of anti-Jo1 autoantibodies in the pathogenesis of ILD in this subset of IIIM/ASS. However, functional studies are needed to confirm this hypothesis. Even though the WHEP domain appears to account for the main reactivity displayed by HisRS, the persistent high anti-HisRS-FL antibody levels over the disease course together with the simultaneous reduction in the antibodies reactivity against the tested HisRS domains and splice variant, suggests that antigenic regions other than the WHEP domain might be present (15) or develop at a later stage.

The administration of rituximab did not result in a homogenous reduction of anti-HisRS reactivity towards the different variant/domains or the full-length protein. This is in contrast to the results from the rituximab in myositis (RIM) trial (33), although differences in methods and time point analysis should be considered when interpreting the results. The persisting anti-HisRS reactivity after rituximab treatment could be explained by the known lack of depletion of memory B cells and plasma cells after rituximab infusion and can contribute to the chronicity of disease and the relapsing disease course seen in many patients with anti-Jo1 autoantibodies (34).

WB experiments were performed to address whether, in addition to the binding of naturally occurring folded HisRS variants, anti-Jo1 antibodies could also recognize non-conformational epitopes within HisRS. The folding of the proteins in ELISA plates was assumed since they were added in native conditions. In addition, to reduce the binding of HisRS proteins directly to the plate and hence also avoiding the risk to unfold the protein, we purposefully produced HisRS variants that retain their native folding and employed a biotinylated Avi-tag that binds to streptavidin coated plates. For the WB experiments, the proteins were added under denatured conditions, thus assuming an unfolded conformation. In line with previous studies $(13,16)$, our results show that anti-Jo1 antibodies

Page $10 / 19$ 
bind conformational independent epitopes, as well as conformational-dependent, with no apparent differences among antibodies available at diagnosis or during follow-up under treatment. Among the five HisRS constructs tested, all denatured HisRS fragments (non-conformational) were recognized by IgG antiJo1 antibodies with the exception of the CD, which could not be detected by WB (17). This could be because the antigenic reactivity of the CD is dependent on the 3-dimensional structure of the domain, whereas within the other domains the autoantibodies recognize both structural as well as linear epitopes.

In this study we have also developed a method to measure an average affinity of autoantibodies against HisRS-FL, using SPR. Strikingly, the majority of anti$\mathrm{Jo}^{+}$patients presented anti-HisRS-FL antibodies with a high-affinity profile already at the time close to diagnosis. Recently, another study showed that individual anti-Jo1 monoclonal antibodies, selected based on somatic hypermutation using single cell isolation and sequencing, also displayed affinities from low $\mathrm{nM} K_{D}$ and below (35). Also, in patients with RA, high affinity circulating autoantibodies (targeting the citrullinated peptide Cit573) have previously been observed (36). Of note, considering that the average affinity is the component being measured, there are some facts to take into consideration; firstly, the analyzed total IgG samples are polyclonal, thereby likely containing a mixture of specific anti-Jo1 autoantibodies of different affinities, most likely present in very different concentrations. Here, an average affinity is measured, and the binding profile of an individual antibody clone within a polyclonal mix may be very different to what we report. For example, it is possible that high affinity antibodies in the sample mask low affinity antibodies. Secondly, the antigen HisRS-FL is a homodimer and this may give rise to an avidity effect, i.e. an apparent increase in affinity. Despite these complicating factors, the analysis was made using the predefined 1:1 Langmuir binding model to get comparable values among the patient samples. Considering the generally high reactivity against all HisRS domains at diagnosis observed in this study, in combination with the fact that high affinity autoantibodies were retrieved from patients with a recent diagnosis of IIM/ASS, one can speculate that affinity maturation of anti-HisRS antibodies through somatic hypermutation together with epitope spreading have already happened before onset of specific symptoms, ultimately leading to diagnosis of IIM/ASS. To confirm this hypothesis, further studies aiming at characterizing anti-Jo1 antibodies before clinical diagnosis are warranted.

A limitation of our study is the use of the Bohan and Peter criteria for classification of IIM as the EULAR/ACR classification criteria (37) for adult and juvenile IIM were not published at the time of patient inclusion. Another limitation is the low number of anti-Jo $1^{+}$patients $(n=19)$. However, since we aimed for an indepth characterization of anti-Jo1 autoantibodies (29), the current study design was a feasible approach. A strength of our study is that the antibody reactivities were analyzed in purified IgG which would diminish interference of other factors in sera that could influence antigen binding. In addition, this was later confirmed to be necessary since some of the reactivities detected using purified IgG from sera, could not be found when using sera. Furthermore, paired BALF-serum samples as well as available longitudinal sera in the anti-Jo $1^{+}$group with clinical data strengthened our observations. Even with the low number of patients included, the results are consistent with a high IgG and IgA reactivity towards the HisRS-FL protein as well as towards the WHEP domain and SV at diagnosis (both in circulation and in the lung) as well as a solid report on the high affinity profile of IgG anti-Jo1 autoantibodies.

\section{Conclusions}

In conclusion, anti-Jo1 autoantibodies of IgG and IgA subclasses from patients with IIM/ASS bind multiple HisRS conformation-dependent and -independent epitopes, already at the time of diagnosis of IIM/ASS and both systemically and locally in the lung. We confirm that the WHEP domain contains the major antiJo1 autoantibody epitope(s) which is strongly overrepresented among anti-Jo1 autoantibodies in circulation and BALF. In addition, we report that anti-HisRSFL IgG autoantibodies exhibit a high affinity profile already at diagnosis, and further show that high anti-HisRS-FL antibody levels in circulation correlate with ILD and arthritis but not with skin and muscle involvement. Our longitudinal results also suggest that anti-HisRS-FL antibody levels could be used as prognostic biomarker for ILD activity in patients with IIM/ASS. Taken together, our data supports the hypothesis that the lung might be a site where aberrant immune activation against HisRS primarily occurs, leading to a systemic inflammatory condition, the anti-synthetase syndrome, with ILD as the main clinical manifestation.

\section{Abbreviations}

aaRS: aminoacyl transfer RNA synthetases; ABD: anti-codon binding domain; ACPA: anti-citrullinated protein/peptide antibodies; ASS: anti-synthetase syndrome; BALF: broncheoalveolar lavage fluid; CD: catalytic domain; CK: creatinine kinase; CRP: protein C-reactive; DAS-28: disease activity score calculated for rheumatoid arthritis; DLCO: diffusion lung capacity for carbon monoxide; DM: dermatomyositis; ELISA: enzyme-linked immunosorbent assay; FEV1: forced expiratory volume in 1 second; FVC: forced vital capacity; HAQ: health assessment questionnaire; HC: healthy controls; HisRS (Jo1): histidyl-transfer RNA synthetase; HisRS-FL: full-length histidyl-transfer RNA synthetase; HRCT: high resolution computed tomography; IIM: idiopathic inflammatory myopathies; IgG/A: immunoglobulin G or A; IBM: inclusion body myositis; ILD: interstitial lung disease; Jo1+: anti-Jo1 positive IIM/ASS patients; Jo1': anti-Jo1 negative IIM/ASS patients; MDDAT: myositis disease activity assessment tool for extra-muscular global assessment; MMT-8: manual muscle testing; NSIP: non-specific interstitial pneumonia; OP: organizing pneumonia; PM: polymyositis; RA: rheumatoid arthritis; SV: splice variant; TLC: total lung capacity; UIP: usual interstitial pneumonia; VAS patient: patient's global disease activity assessment; VAS physician: physician's global disease activity assessment; VC: vital capacity; WB: western blot.

\section{Declarations}

\section{Ethics approval and consent to participate}

This study was approved by the Ethics Committee at Karolinska Institutet, Sweden. All patients gave written informed consent.

\section{Consent for publication}

All authors read and approved the manuscript. 
All data generated or analyzed during this study are included in this published article and its supplementary information files.

\section{Competing interest}

Dr. Lundberg has received a research grant from Bristol Myers Squibb and from Astra Zeneca and has served on advisory board of Corbus Pharmaceuticals, Inc., Argenx, Kezaar, Octapharma, Orphazyme, EMD Serono Research \& Development Institute, and Janssen. I Lundberg has stock shares in Roche and Novartis. The other authors declare no competing interests.

\section{Funding}

This study was funded by: Association Française contre les Myopathies (AFM-Téléthon); Reumatikerförbundet; Karolinska Institutet's Foundation Grants for Rheumatology Research; Ulla and Gustaf af Uggla Foundation; The Swedish Heart-Lung foundation; The Myositis Association; The Swedish Research Council no 2016-01254; The Swedish Rheumatism Association; King Gustaf V 80 Year Foundation; Konung Gustaf V:s och Drottning Victorias Frimurarestiftelse; Funds at the Karolinska Institutet (KID) and Region Stockholm (ALF project); EU/EFPIA Innovative Medicines Initiative Joint Undertaking (ULTRA-DD grant ${ }^{\circ}$ 115766) and the EU/EFPIA/OICR/McGill/KTH/Diamond Innovative Medicines Initiative 2 Joint Undertaking (EUbOPEN grant $\left.n^{\circ} 875510\right)$. Disclaimer: This communication reflects the views of the authors and neither IMI nor the European Union, EFPIA or any Associated Partners are liable for any use that may be made of the information contained herein.

\section{Authors' contributions}

AN, CP, IEL and CFC designed the study. CFC prepared the Jo1 affinity column, performed the ELISAs and WBs, interpreted and analyzed the data, and wrote the first draft of the manuscript. CFC together with NR purified the antibodies. CP performed, analyzed, and interpreted the SPR experiments. HP contributed to the analysis and interpretation of SPR experiments. AN collected clinical data and assisted on the correlations between the experimental results and clinical manifestations. EW and SG produced all recombinant HisRS proteins. CP, NR, EVG and AGF assisted CFC running the ELISAs. MF and JG collected BALF. SL ran the multi-variate analysis. PJJ critically reviewed the paper and helped interpreting the results. AN, CP, IEL and CFC wrote the final version of the manuscript. All authors read and gave relevant input on the manuscript.

\section{Acknowledgements}

Julia Norkko for handling of serum biobank. Benita Engvall for handling of BALF biobank. Francesco Bonomi for assistance withdrawing clinical information.

\section{References}

1. Johnson C, Pinal-Fernandez I, Parikh R, Paik J, Albayda J, Mammen AL, et al. Assessment of Mortality in Autoimmune Myositis With and Without Associated Interstitial Lung Disease. Lung. 2016;194(5):733-7.

2. Dobloug GC, Svensson J, Lundberg IE, Holmqvist M. Mortality in idiopathic inflammatory myopathy: results from a Swedish nationwide population-based cohort study. Ann Rheum Dis. 2018;77(1):40-7.

3. Marguerie C, Bunn CC, Beynon HL, Bernstein RM, Hughes JM, So AK, et al. Polymyositis, pulmonary fibrosis and autoantibodies to aminoacyl-tRNA synthetase enzymes. Q J Med. 1990;77(282):1019-38.

4. Brouwer R, Hengstman GJ, Vree Egberts W, Ehrfeld H, Bozic B, Ghirardello A, et al. Autoantibody profiles in the sera of European patients with myositis. Ann Rheum Dis. 2001;60(2):116-23.

5. Hamaguchi Y, Fujimoto M, Matsushita T, Kaji K, Komura K, Hasegawa M, et al. Common and distinct clinical features in adult patients with antiaminoacyl-tRNA synthetase antibodies: heterogeneity within the syndrome. PLoS One. 2013;8(4):e60442.

6. Betteridge Z, Tansley S, Shaddick G, Chinoy H, Cooper RG, New RP, et al. Frequency, mutual exclusivity and clinical associations of myositis autoantibodies in a combined European cohort of idiopathic inflammatory myopathy patients. J Autoimmun. 2019;101:48-55.

7. Richards TJ, Eggebeen A, Gibson K, Yousem S, Fuhrman C, Gochuico BR, et al. Characterization and peripheral blood biomarker assessment of anti-Jo-1 antibody-positive interstitial lung disease. Arthritis Rheum. 2009;60(7):2183-92.

8. Kwon NH, Fox PL, Kim S. Aminoacyl-tRNA synthetases as therapeutic targets. Nat Rev Drug Discov. 2019;18(8):629-50.

9. Xu Z, Wei Z, Zhou JJ, Ye F, Lo WS, Wang F, et al. Internally deleted human tRNA synthetase suggests evolutionary pressure for repurposing. Structure. 2012;20(9):1470-7.

10. Zhou JJ, Wang F, Xu Z, Lo WS, Lau CF, Chiang KP, et al. Secreted histidyl-tRNA synthetase splice variants elaborate major epitopes for autoantibodies in inflammatory myositis. J Biol Chem. 2014;289(28):19269-75.

11. Lo WS, Gardiner E, Xu Z, Lau CF, Wang F, Zhou JJ, et al. Human tRNA synthetase catalytic nulls with diverse functions. Science. 2014;345(6194):328-32.

12. Adams RA, Fernandes-Cerqueira C, Notarnicola A, Mertsching E, Xu Z, Lo WS, et al. Serum-circulating His-tRNA synthetase inhibits organ-targeted immune responses. Cell Mol Immunol. 2021;18(6):1463-75.

13. Ramsden DA, Chen J, Miller FW, Misener V, Bernstein RM, Siminovitch KA, et al. Epitope mapping of the cloned human autoantigen, histidyl-tRNA synthetase. Analysis of the myositis-associated anti-Jo-1 autoimmune response. J Immunol. 1989;143(7):2267-72.

14. Miller FW, Waite KA, Biswas T, Plotz PH. The role of an autoantigen, histidyl-tRNA synthetase, in the induction and maintenance of autoimmunity. Proc Natl Acad Sci U S A. 1990;87(24):9933-7. 
15. Miller FW, Twitty SA, Biswas T, Plotz PH. Origin and regulation of a disease-specific autoantibody response. Antigenic epitopes, spectrotype stability, and isotype restriction of anti-Jo-1 autoantibodies. J Clin Invest. 1990;85(2):468-75.

16. Raben N, Nichols R, Dohlman J, McPhie P, Sridhar V, Hyde C, et al. A motif in human histidyl-tRNA synthetase which is shared among several aminoacyltRNA synthetases is a coiled-coil that is essential for enzymatic activity and contains the major autoantigenic epitope. J Biol Chem. 1994;269(39):2427783.

17. Martin A, Shulman MJ, Tsui FW. Epitope studies indicate that histidyl-tRNA synthetase is a stimulating antigen in idiopathic myositis. FASEB J. 1995;9(12):1226-33.

18. Levine SM, Raben N, Xie D, Askin FB, Tuder R, Mullins M, et al. Novel conformation of histidyl-transfer RNA synthetase in the lung: the target tissue in Jo-1 autoantibody-associated myositis. Arthritis Rheum. 2007;56(8):2729-39.

19. Galindo-Feria AS, Albrecht I, Fernandes-Cerqueira C, Notarnicola A, James EA, Herrath J, et al. Proinflammatory Histidyl-Transfer RNA Synthetase-Specific CD4 + T Cells in the Blood and Lungs of Patients With Idiopathic Inflammatory Myopathies. Arthritis Rheumatol. 2020;72(1):179-91.

20. Bohan A, Peter JB. Polymyositis and Dermatomyositis (first of two parts). N Engl J Med. 1975;292(7):344-7.

21. Bohan A, Peter JB. Polymyositis and dermatomyositis (second of two parts). N Engl J Med. 1975;292(8):403-7.

22. Griggs RC, Askanas V, DiMauro S, Engel A, Karpati G, Mendell JR, et al. Inclusion body myositis and myopathies. Ann Neurol. 1995;38(5):705-13.

23. Connors GR, Christopher-Stine L, Oddis CV, Danoff SK. Interstitial lung disease associated with the idiopathic inflammatory myopathies: what progress has been made in the past 35 years? Chest. 2010;138(6):1464-74.

24. Rider LG, Werth VP, Huber AM, Alexanderson H, Rao AP, Ruperto N, et al. Measures of adult and juvenile dermatomyositis, polymyositis, and inclusion body myositis: Physician and Patient/Parent Global Activity, Manual Muscle Testing (MMT), Health Assessment Questionnaire (HAQ)/Childhood Health Assessment Questionnaire (C-HAQ), Childhood Myositis Assessment Scale (CMAS), Myositis Disease Activity Assessment Tool (MDAAT), Disease Activity Score (DAS), Short Form 36 (SF-36), Child Health Questionnaire (CHQ), physician global damage, Myositis Damage Index (MDI), Quantitative Muscle Testing (QMT), Myositis Functional Index-2 (FI-2), Myositis Activities Profile (MAP), Inclusion Body Myositis Functional Rating Scale (IBMFRS), Cutaneous Dermatomyositis Disease Area and Severity Index (CDASI), Cutaneous Assessment Tool (CAT), Dermatomyositis Skin Severity Index (DSSI), Skindex, and Dermatology Life Quality Index (DLQI). Arthritis Care Res (Hoboken). 2011;63 Suppl 11:S118-57.

25. Travis WD, Costabel U, Hansell DM, King TE Jr, Lynch DA, Nicholson AG, et al. An official American Thoracic Society/European Respiratory Society statement: Update of the international multidisciplinary classification of the idiopathic interstitial pneumonias. Am J Respir Crit Care Med. 2013;188(6):733-48.

26. van Gestel AM, Prevoo ML, van 't Hof MA, van Rijswijk MH, van de Putte LB, van Riel PL. Development and validation of the European League Against Rheumatism response criteria for rheumatoid arthritis. Comparison with the preliminary American College of Rheumatology and the World Health Organization/International League Against Rheumatism Criteria. Arthritis Rheum. 1996;39(1):34-40.

27. Preger C, Wigren E, Ossipova E, Marks C, Lengqvist J, Hofstrom C, et al. Generation and validation of recombinant antibodies to study human aminoacyltRNA synthetases. J Biol Chem. 2020;295(41):13981-93.

28. Ossipova E, Cerqueira CF, Reed E, Kharlamova N, Israelsson L, Holmdahl R, et al. Affinity purified anti-citrullinated protein/peptide antibodies target antigens expressed in the rheumatoid joint. Arthritis Res Ther. 2014;16(4):R167.

29. Fernandes-Cerqueira C, Renard N, Notarnicola A, Wigren E, Graslund S, Zubarev RA, et al. Patients with anti-Jo1 antibodies display a characteristic IgG Fcglycan profile which is further enhanced in anti-Jo1 autoantibodies. Sci Rep. 2018;8(1):17958.

30. La Corte R, Lo Mo Naco A, Locaputo A, Dolzani F, Trotta F. In patients with antisynthetase syndrome the occurrence of anti-Ro/SSA antibodies causes a more severe interstitial lung disease. Autoimmunity. 2006;39(3):249-53.

31. Vancsa A, Csipo I, Nemeth J, Devenyi K, Gergely L, Danko K. Characteristics of interstitial lung disease in SS-A positive/Jo-1 positive inflammatory myopathy patients. Rheumatol Int. 2009;29(9):989-94.

32. Bauhammer J, Blank N, Max R, Lorenz HM, Wagner U, Krause D, et al. Rituximab in the Treatment of Jo1 Antibody-associated Antisynthetase Syndrome: Anti-Ro52 Positivity as a Marker for Severity and Treatment Response. J Rheumatol. 2016;43(8):1566-74.

33. Aggarwal R, Oddis CV, Goudeau D, Koontz D, Qi Z, Reed AM, et al. Autoantibody levels in myositis patients correlate with clinical response during B cell depletion with rituximab. Rheumatology. 2016;55(6):991-9.

34. Kamburova EG, Koenen HJ, Borgman KJ, ten Berge IJ, Joosten I, Hilbrands LB. A single dose of rituximab does not deplete B cells in secondary lymphoid organs but alters phenotype and function. Am J Transplant. 2013;13(6):1503-11.

35. Burman L, Chong YE, Duncan S, Klaus A, Rauch K, Hamel K, et al. Isolation of monoclonal antibodies from anti-synthetase syndrome patients and affinity maturation by recombination of independent somatic variants. MAbs. 2020;12(1):1836718.

36. Fernandes-Cerqueira C, Ossipova E, Gunasekera S, Hansson M, Mathsson L, Catrina Al, et al. Targeting of anti-citrullinated protein/peptide antibodies in rheumatoid arthritis using peptides mimicking endogenously citrullinated fibrinogen antigens. Arthritis Res Ther. 2015;17(1):155.

37. Bottai M, Tjarnlund A, Santoni G, Werth VP, Pilkington C, de Visser M, et al. EULAR/ACR classification criteria for adult and juvenile idiopathic inflammatory myopathies and their major subgroups: a methodology report. RMD Open. 2017;3(2):e000507.

\section{Figures}


A

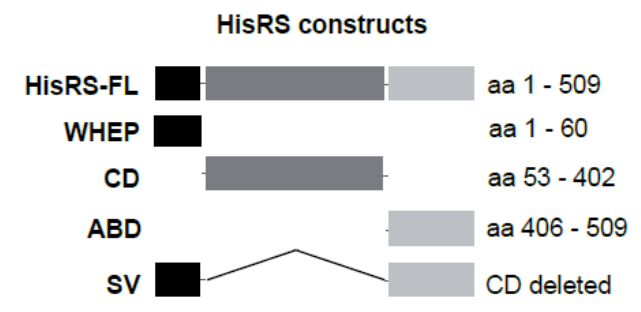

C
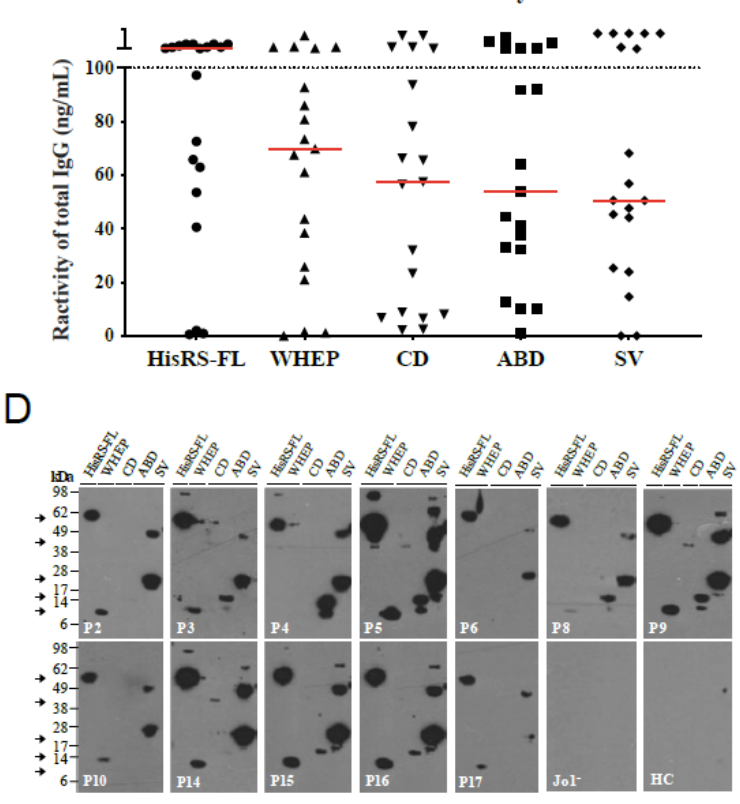

$B$

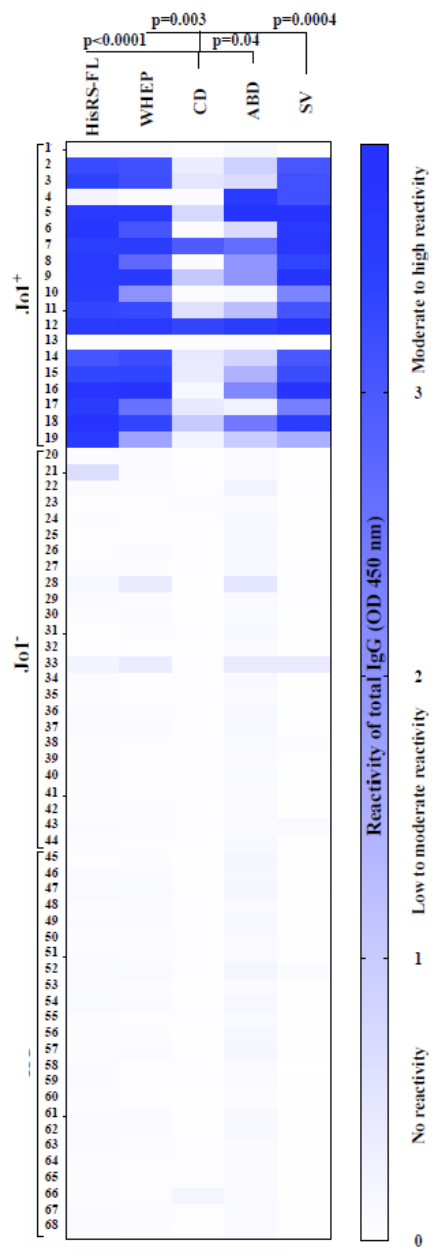

\section{Figure 1}

Anti-Jo1 autoantibodies display high reactivity against HisRS full-length/variant/domains at IIM/ASS diagnosis. (A) Schematic figure of HisRS versions used in the experiments. (B) The reactivity towards HisRS-FL, WHEP, CD, ABD, and SV as conformational epitopes was measured by ELISA in IgG purified from serum of 19 anti-Jo1+, 25 anti-Jo1-, and 24 healthy controls (HC). High reactivity (OD $450 \mathrm{~nm}$ ) corresponds to strong blue color. (C) Anti-HisRS reactivity ( $\mathrm{ng} / \mathrm{mL}$ concentration) of anti-Jo1+ total IgG against HisRS-FL, WHEP, CD, ABD, and SV. Antibody titers were calculated using a standard curve (Supplementary Figure 3B) and titers were measured in the linear range between 5 and $100 \mathrm{ng} / \mathrm{mL}$. Median values for each antigen are indicated by the red line. (D) Anti-Jo1 reactivity against denatured HisRS antigens was addressed by WB in IgG purified from serum of 12 representative anti-Jo1+, 1 anti-Jo1-, and 1 HC. Strong band intensity denotes higher anti-Jo1 reactivity. Arrows indicate the molecular weight of the different HisRS variants (Supplementary Table 2, Supplementary Figure 1). Some patients also showed an additional band, corresponding to the HisRS dimer. Kruskal-Wallis test in (B) was applied. P<0.05 was assumed as significantly different. 
A

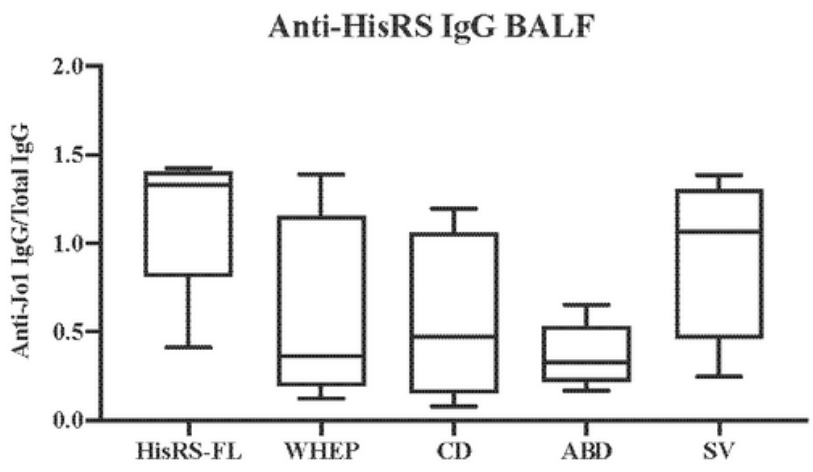

C

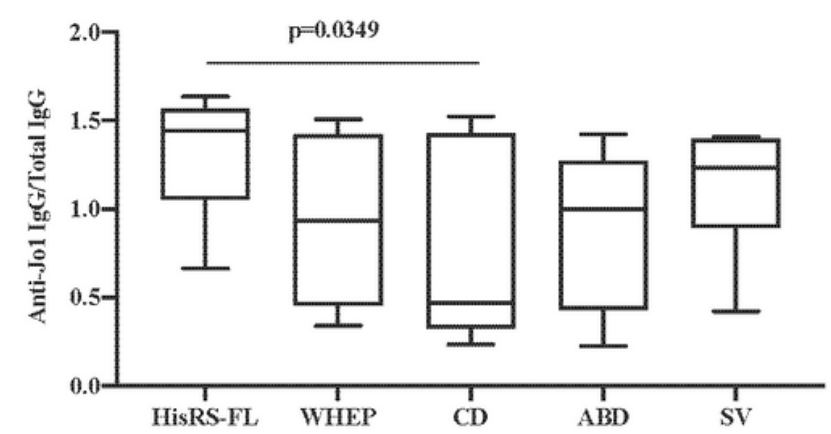

$\mathrm{B}$

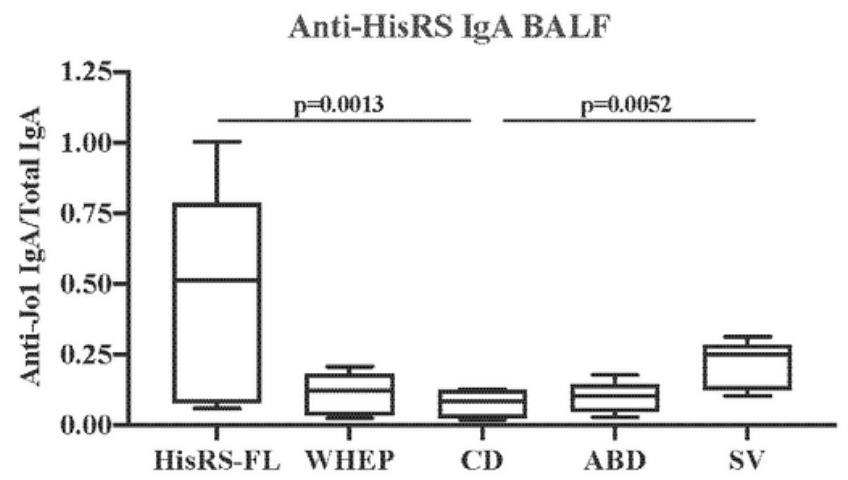

D

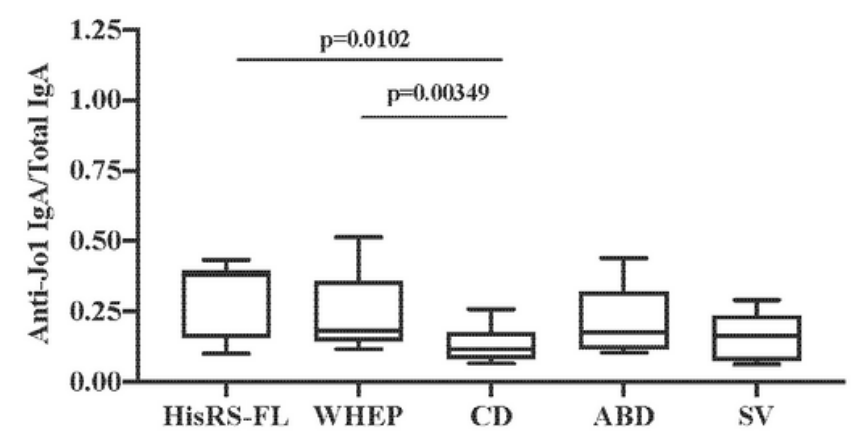

Figure 2

Reactivity of serum and BALF-derived anti-Jo1 antibodies. (A, C) Anti-Jo1 IgG and (B, D) IgA reactivity in BALF and paired serum were measured by ELISA in 6 anti-Jo1+ patients. Autoantibody levels were normalized to total values of IgG and IgA (Y-axis). Friedman's tests corrected for multiple comparisons by Dunn's test was applied. $\mathrm{P}<0.05$ was assumed as significantly different. No significant differences were found in $(A)$. 
A
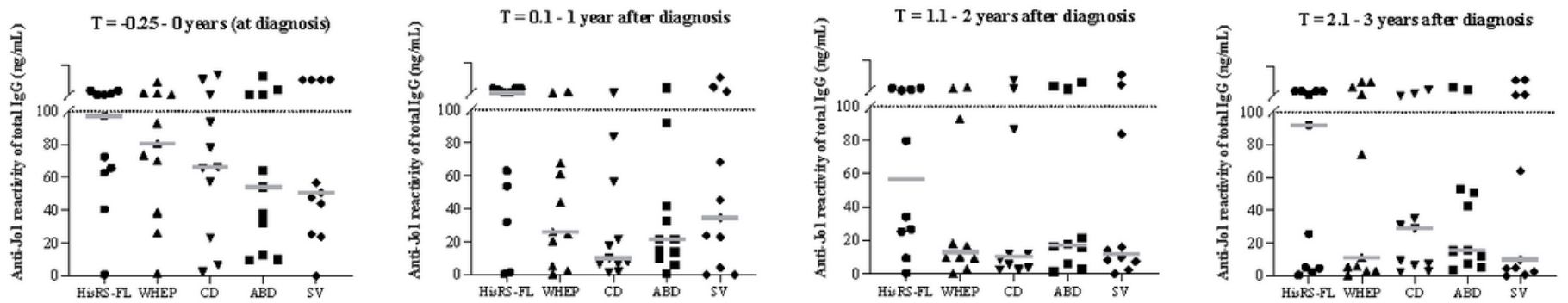

B
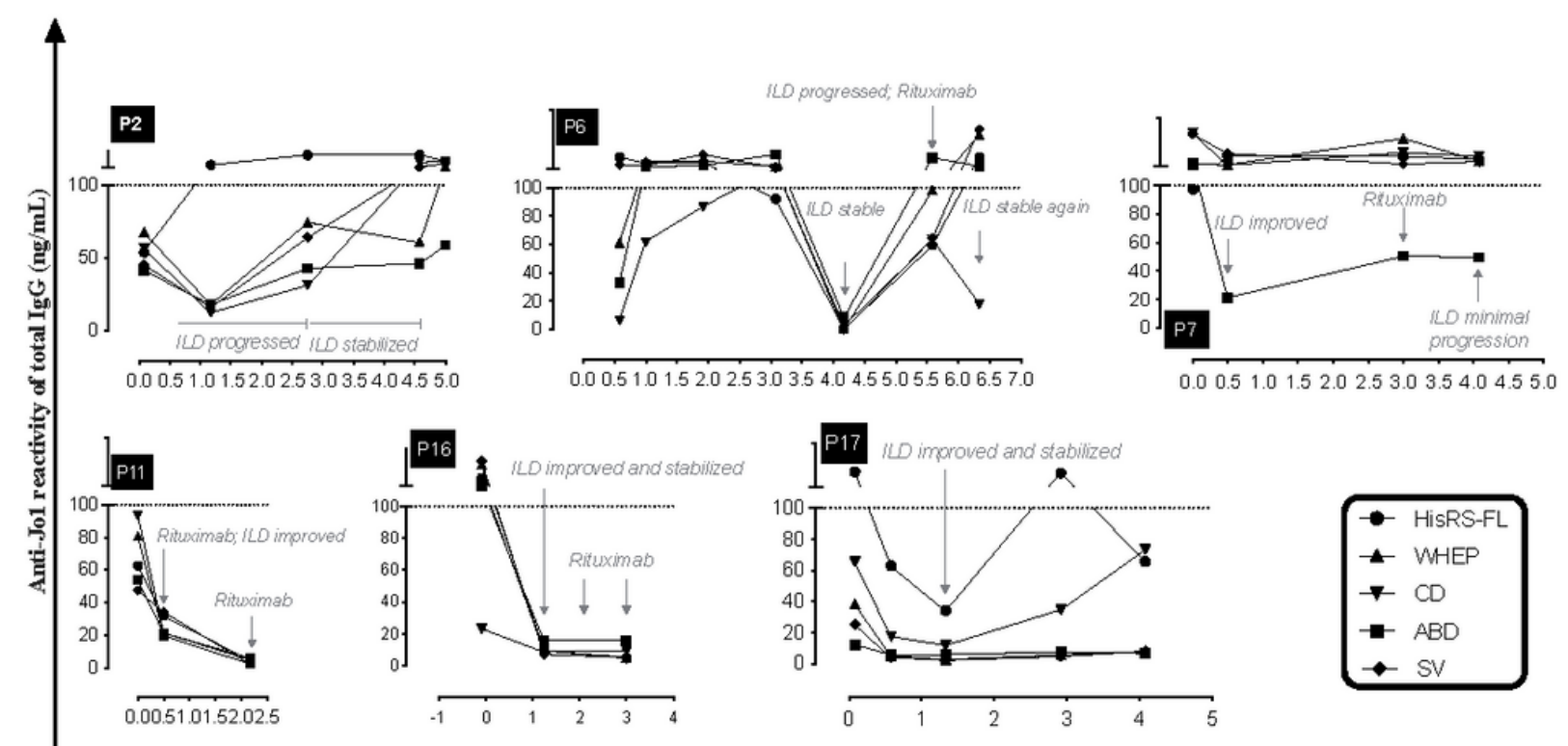

Disease duration (years)

\section{Figure 3}

Reactivity of anti-Jo1 autoantibodies towards HisRS variant and domains decreases over time but remains high against HisRS-FL. (A) Reactivity against HisRS-FL, -splice variant (SV), and -domains (WHEP, CD and ABD) displayed by total IgG purified from the first available anti-Jo1+ sera close to diagnosis ( $T=$ $-0.25-0$ years), $T=0.1-1, T=1.1-2$, and $T=2.1-3$ years after diagnosis. Additional graphs displaying anti-Jo1 reactivity against HisRS-FL, variant, and domains are displayed in Supplementary Figure 7 upper panel. (B) Anti-Jo1 reactivity of 6 anti-Jo1+ patients (P2, P6, P7, P11, P16, P17) displayed by total IgG purified from sera collected longitudinally. Y-axis represents anti-Jo1 antibody levels against HisRS, measured in the total IgG fraction isolated from anti-Jo1+ IIM/ASS sera. X-axis represents disease duration in years. Grey italic sentences provide information on rituximab introduction and/or follow-up on interstitial lung disease. Concentration $(\mathrm{ng} / \mathrm{mL})$ of anti-Jo1 antibodies was calculated based on a standard curve derived from anti-Jo1 IgG isolated from a sera pool of 38 anti-Jo1+ IIM/ASS individuals, titers were measured in the linear range between 5 and $100 \mathrm{ng} / \mathrm{mL}$ (Supplementary Figure 3B (29)). The letter P (Patient) followed by a number in each graph represents an anti-Jo1+ IIM/ASS individual. 
A

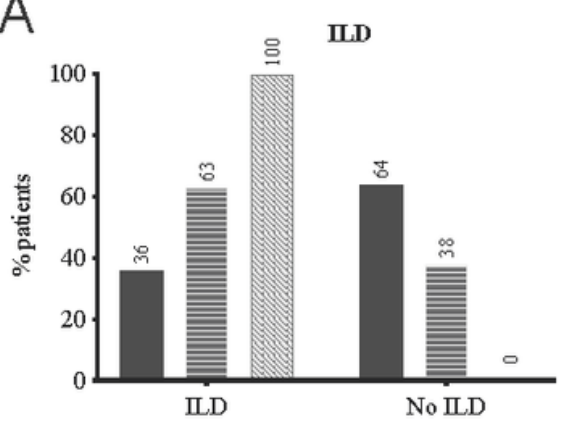

D

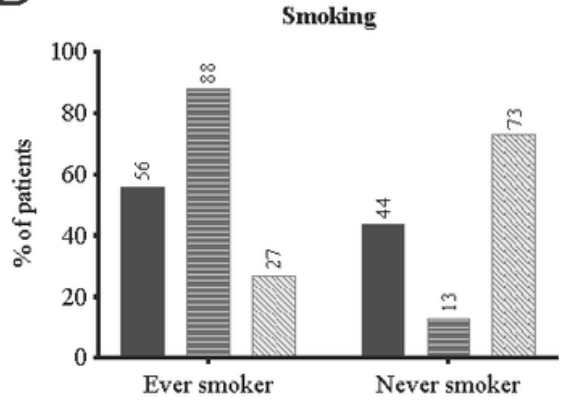

B

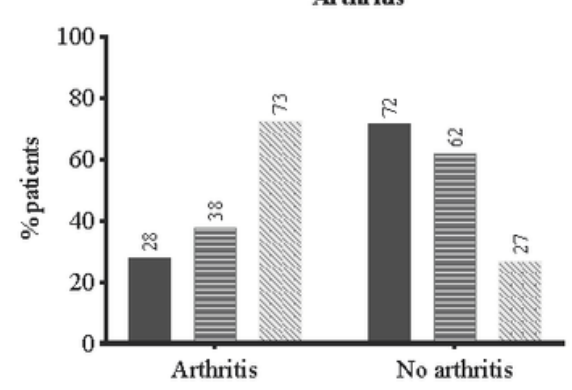

E

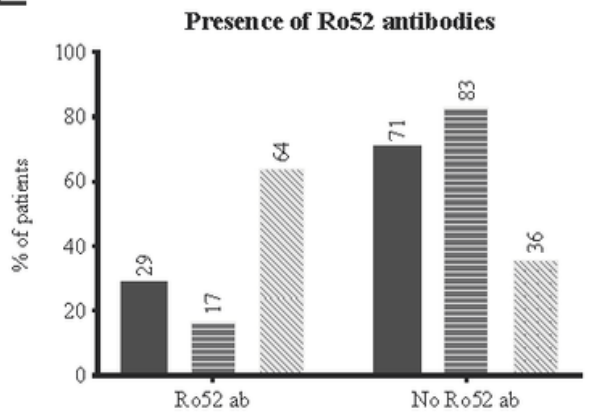

C

Skin rash

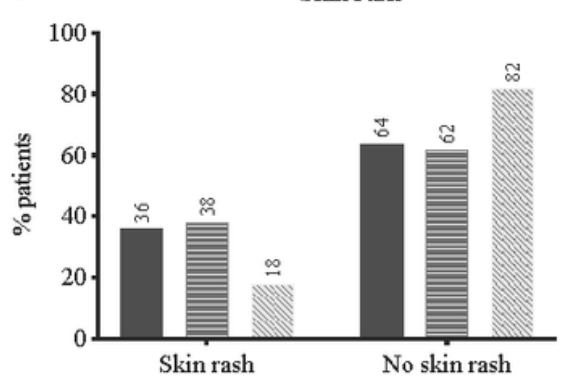

Anti-HisRS-FL

$\equiv$ Anti-HisRS-FL ${ }^{+}+(0.5-100 \mathrm{ng} / \mathrm{mL})$

Anti-HisRS-FL ${ }^{+}+(>100 \mathrm{ng} / \mathrm{mL})$

\section{Figure 4}

IIM/ASS patients diagnosed with ILD and arthritis and less skin involvement harbor more reactive anti-Jo1 autoantibodies. (A-B-C-D-E) Percentage of IIM/ASS patients distributed according to anti-HisRS full-length (HisRS-FL) reactivity levels, clinical diagnosis and clinical manifestations. The anti-HisRS-FL reactivity displayed was measured in total anti-Jo1+ IgG purified from serum. Numbers on top of the bars represent the percentage of patients in the group. HisRS-FL-; anti-Jo1 IIM/ASS negative patients; Anti-HisRS-FL+ +, anti-Jo1 IIM/ASS positive patients with low to moderate reactivity (0.5 - $100 \mathrm{ng} / \mathrm{mL})$; Anti-HisRS-FL+ ++, anti-Jo1 IIM/ASS positive patients with high HisRS reactivity (> $100 \mathrm{ng} / \mathrm{mL})$. Antibody titers were calculated from OD (450 $\mathrm{nm})$ using the standard curve in Supplementary Figure 3B. IIM, idiopathic inflammatory myopathies; ILD, interstitial lung disease; ASS, anti-synthetase syndrome. Statistical differences among groups are displayed in Supplementary Table 3. 

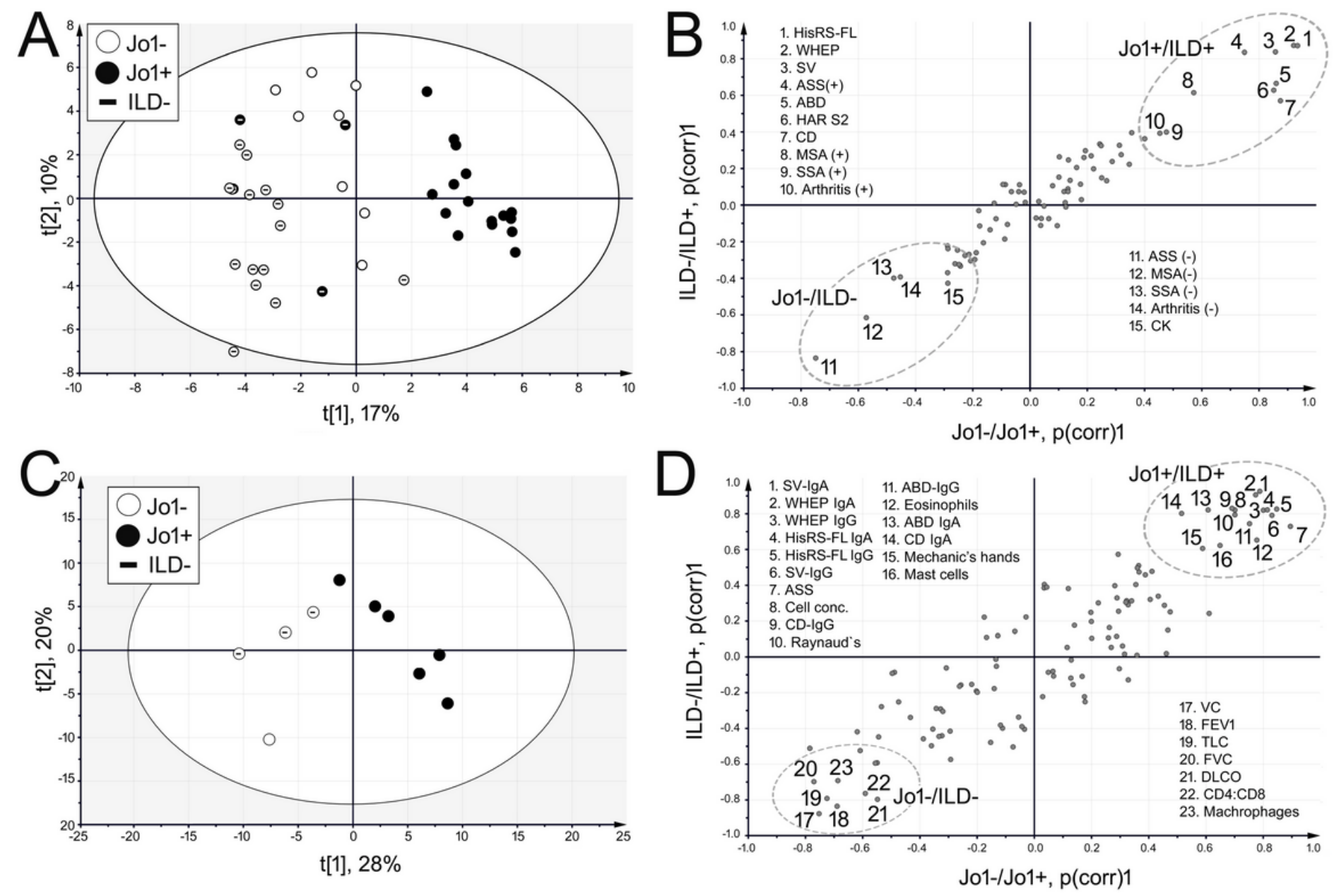

Figure 5

Multivariate data analysis shows a strong correlation between anti-Jo1 autoantibody reactivity profile, ILD and arthritis, and poor pulmonary function. (A) Principal component analysis (PCA) scores plot of the baseline data obtained from the patients (cohort 1, n=44) which demographics are listed in Table 1. (B) Shared and Unique Structures (SUS) plot obtained from two OPLS-DA models: 1) distinguishing the patients according to anti-Jo1 status (predictive component: $\mathrm{R} 2=0.73, \mathrm{Q} 2=0.59$ ), and 2) distinguishing the patients according to ILD status (predictive component: $\mathrm{R} 2=0.74, \mathrm{Q} 2=0.60$ ). The data processed is the same as in the PCA plot in cohort 1 (A). The factors that correlated most strongly with anti-Jo1+/ILD+ as well as anti-Jo1-/ILD- are highlighted. (C) PCA scores plot of the baseline data obtained from the patients (cohort 2, $n=10$ ) which demographics are listed in Supplementary Table 1. (D) Shared and Unique Structures (SUS) plot obtained from two OPLS-DA models: 1) distinguishing the patients according to Jo1 status (predictive component: R2=0.86, Q2=0.61) and 2) distinguishing the patients according to ILD status (predictive component: R2=0.76, Q2=0.43). The data processed is the same as in the PCA plot of cohort 2 in (C). 

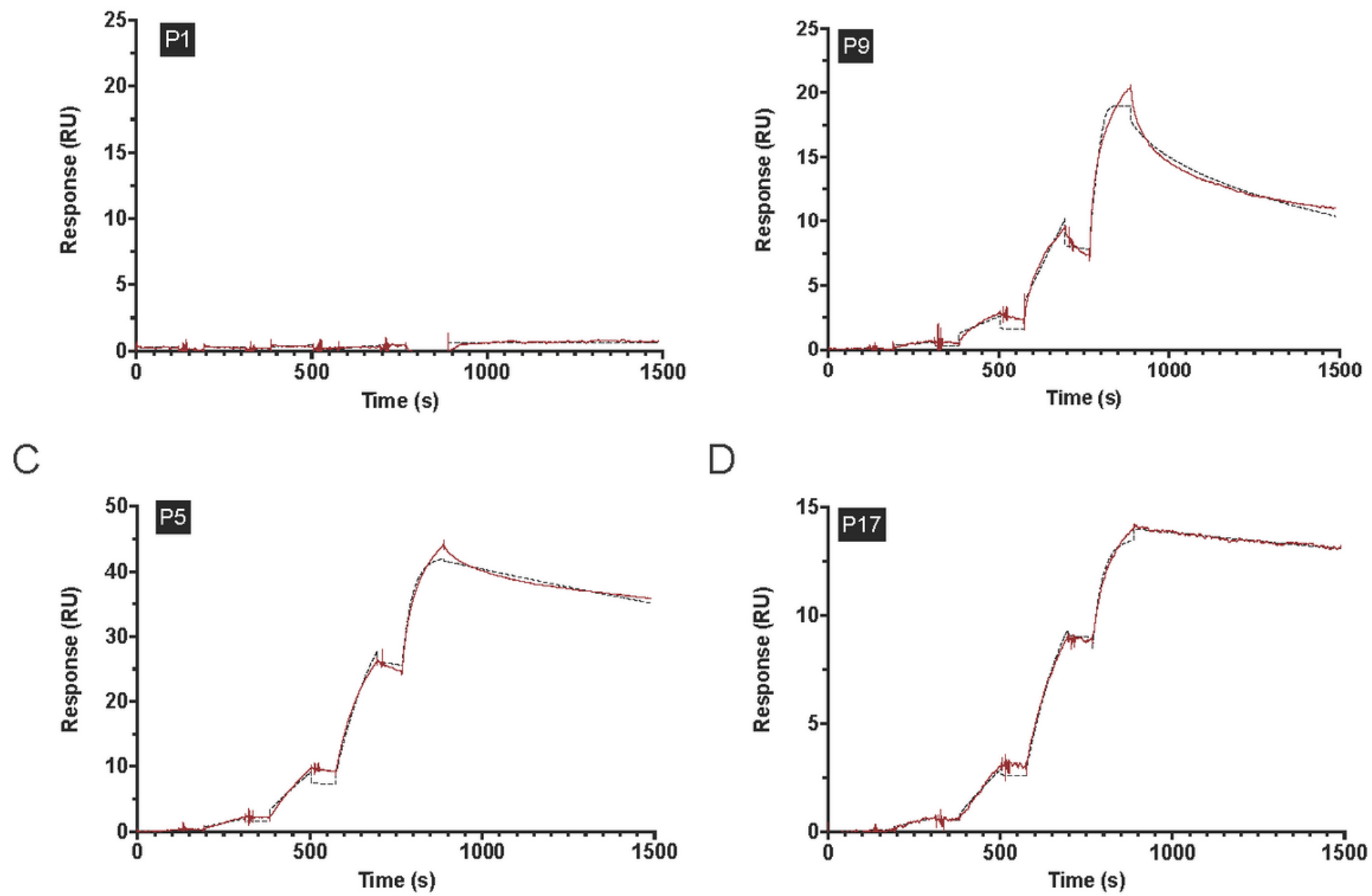

Figure 6

Affinity binding profiles against HisRS-FL for IgG purified from sera at first available sample close to diagnosis measured by SPR in single cycle kinetics mode. Sensorgrams showing the obtained signal in response units ( $R U, y$-axis) over time (seconds, s, $x$-axis) from four representative patients ( $P 1$, $P 5$, $P$ 9, and $P 17)$. (A) Three patients did not show any binding in SPR, as indicated by a flat line (same observation for P4 and P13, data not shown). (B) P9 is an example of a patient having a biphasic off-rate (Avekd). This sort of heterogeneity was also observed for 6 other patients (P3, P7, P11, P12, P16, and P18). (C, D) A slower and more homogenous off-rate (Avekd < 0.0005 s-1) was observed for P5 and P17 (as well as P2, P8, P14, P15, P19), representing the other group ( $n=7$ ) of patients. The biphasic binding profiles observed in approximately half the patients $(n=7)$ could be explained by an avidity effect caused by the dimeric form of the antigen but can also be the result of the polyclonal nature of the patient sample and, thus, containing antibodies of different off-rates. Red lines represent the measured data values and dashed black lines represent the fit of the curve using 1:1 Langmuir binding model. To distinguish the average values reported herein from traditionally reported affinity (KD) and dissociation rate constants (kd), we use the nomenclature AveKD and Avekd.

\section{Supplementary Files}

This is a list of supplementary files associated with this preprint. Click to download.

- Notarnicolaetal.SupplementaryManuscript20210701.docx 